\title{
Physicochemistry aspects on frictional interfaces
}

\author{
Meirong CAI, Qiangliang YU, Feng ZHOU*, Weimin LIU \\ State Key Laboratory of Solid Lubrication, Lanzhou Institute of Chemical Physics, Chinese Academy of Sciences, Lanzhou 730000, China \\ Received: 22 June 2017 / Revised: 22 August 2017 / Accepted: 02 September 2017 \\ (C) The author(s) 2017. This article is published with open access at Springerlink.com
}

\begin{abstract}
Friction exists wherever relative motion occurs and is the main source of energy consumption. Lubrication plays a significant role in improving fuel efficiency, reducing emissions, and prolonging the service life of machines. Surface interactions between two moving solid surfaces or the flow of a fluid (and/or environment) on a solid surface are the primary causes of friction. Apart from the mechanical design of moving parts, surface physicochemistry is of crucial importance to lubrication. This review deals with the frontier research on controlling friction and lubrication, highlights the importance of physicochemistry aspects, and enumerates the state-of-the-art chemistry solutions to tribological issues. It aims at inspiring talented young scientists from different fields to make significant contributions to the area.
\end{abstract}

Keywords: physicochemistry; friction interfaces; surface adsorption; tribochemistry

\section{Introduction}

Tribology is the science of the interface of surfaces in relative motion, and primarily deals with friction, lubrication, and wear. Friction consumes a large amount of energy, which along with wear-related energy and material losses, costs about $5 \%-7 \%$ of the gross national product in most industrialized nations [1]. Thus, a reduction in friction will benefit the economic well-being of all nations. Although in many cases, such as in brake systems and roads, high friction is required, this review will basically focus on friction reduction (lubrication) of sliding surfaces and antiwear of materials. Lubrication aims to reduce the friction on a surface with a bearing capacity and tangential shear strength, and it has become one of the core techniques for the purpose of reducing carbon emissions and saving energy [2].

Liquid lubrication can be described by the wellknown Stribeck curve, as depicted in Fig. 1. The friction coefficient varies with $Z \cdot N / P$, where $Z$ is viscosity of the liquid, $N$ is sliding velocity, and $P$ is applied load. In hydrodynamic and elastohydrodynamic lubrication (EHD or EHL) lubrication, the sliding surfaces are completely separated by a continuous film of lubricating fluid. In that scenario, the friction coefficient is directly related to the fluid viscosity. Mixed lubrication occurs when the velocity decreases and the asperities of the rough surfaces start to touch each other. In this case, part of the load is carried by the direct contact between asperities, thus leading to increased friction. Mixed lubrication is a transition regime between boundary lubrication and EHL, and contains characteristics of both of them. Boundary lubrication is a condition in which the lubricant film becomes too thin to support a substantial part of the load, and the asperities come into a sufficiently close contact to cause "stick-slip" break-off of some of them. The main task of a tribological study is to reduce the friction coefficient at all lubrication regimes. As it will be seen, the interface interaction between lubrication medium and frictional materials plays an important role in friction reduction. Optimization of the lubrication condition highly depends on the physical and chemical properties of the solid-liquid interface. In the region of boundary lubrication, the friction coefficient becomes significantly large, and thus, it requires an effective

* Corresponding author: Feng ZHOU, E-mail: zhouf@licp.cas.cn 


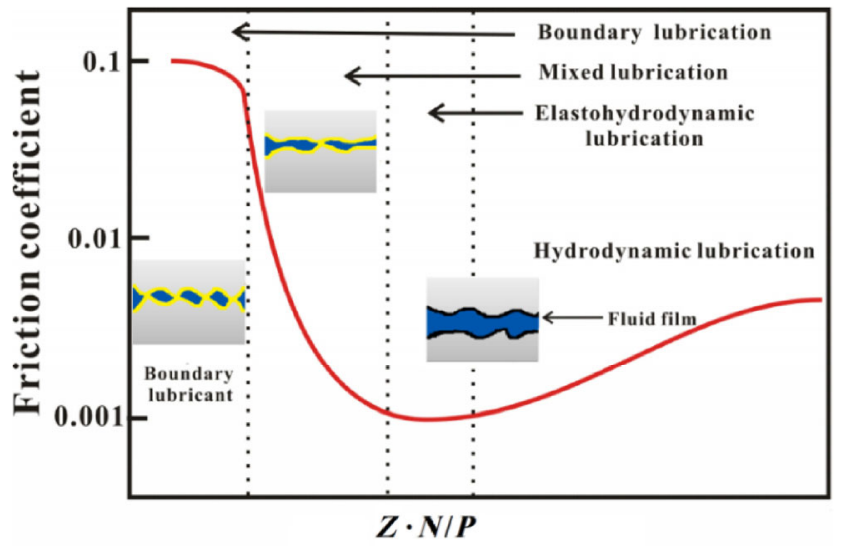

Fig. 1 Stribeck curve during liquid lubrication.

lubrication technology for reducing friction, such as friction modifier, extreme pressure and antiwear agent, and viscosity optimization. The following sections will discuss in detail the research frontier of the boundary and mixed lubrication practice, and their relevant solutions regarding physics, chemistry, and materials.

\section{Friction reduction through surface adsorption or surface grafts}

In hydrodynamic lubrication, the sliding pairs are separated by a liquid film that is self-generated by the moving surfaces by drawing the lubricant into the wedge, and is formed by the bounding surfaces at a high enough velocity to generate the required pressure to completely separate the surfaces and support the applied load. When the load is further increased or the speed of relative motion is reduced, the fluid thin film cannot be effectively formed to bear a high load, and lubrication will evolve into elastohydrodynamic or boundary lubrication (Fig. 1). This is a condition in which the lubricant film becomes too thin to provide total separation, and where the asperities come into close contact and deform or break-off [3]. It is well known that non-wetting will not benefit friction reduction under enhanced loading, because liquids would be very easily squeezed out of the contact area when load is applied (Fig. 2(a)). Lubricating oils have to wet the surface to form effective lubricating films (Fig. 2(b)). Meanwhile, liquids must have sufficient viscosity to form a hydrodynamic pressure film between the sliding pairs. Therefore, oils (mineral oils, polyolefins, polyether, perfluoro-

polyether, polyol esters, etc.) are widely used in practice, because of their relative high viscosity, while water has very low viscosity and can hardly bear high load.

\subsection{Base oils from low polarity to high polarity}

The above listed oils are widely used in industry. However, their lubricating performance and loadcarrying capacities are not satisfactory at severe conditions, such as high loads or low sliding velocities. Besides the improvement of dynamic viscosities of base oils by molecular design, the lubricating and antiwear properties are normally ameliorated by enhancing the surface affinity of lubricants (Fig. 3(a)). Increasing the polarity of mineral oils and polyolefins to polyether and polyol esters lubricants has led to increased load carrying capacity, because the latter oils have stronger interaction with the frictional surfaces. The drawback of a high polarity structure is the easy attack by external triggers (environment, friction practice). Polyethers are prone to degrade and polyol esters are liable to hydrolysis.
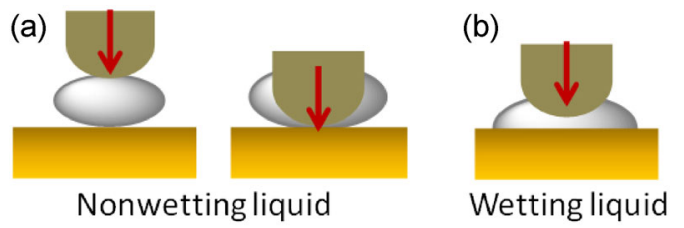

Fig. 2 Stability of liquid upon increasing load. Liquids are prone to be squeezed out of the contact area of sliding, leading to dry friction. Good wetting ability is required to form a stable lubricating film.

(a)

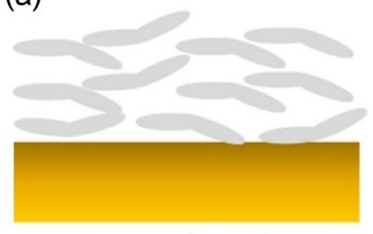

Less polar oils

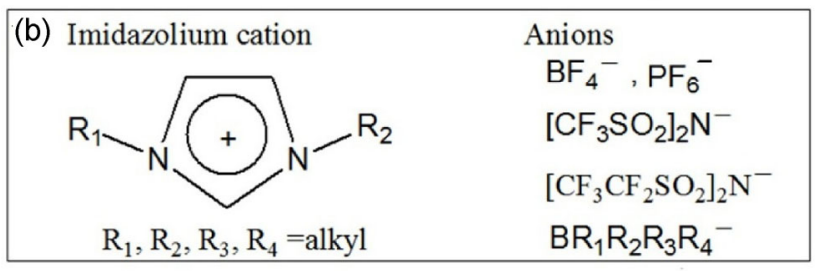

Fig. 3 (a) Diagram of lubricating oil interaction with frictional surface: from non/less polarity to high polarity oils. (b) Representative cation and anions that constitute ionic liquids. 
A recent successful example in novel high polarity lubricating oils is the design of ionic liquids (ILs) lubricants [4-9]. ILs are ion pairs of cations and anions (Fig. 3(b) shows the structure of imidazolium based ILs), which remain with good mobility even at low temperatures, and maintain stability at high temperatures (some of them have over $350{ }^{\circ} \mathrm{C}$ decomposition temperature). The high polarity of ILs molecules provides them with high affinity to the friction surface, the main factor leading to good lubricity and load carrying capacity (over 5 GPa vs. $3 \mathrm{GPa}$ of conventional oils). Forsyth et al. have studied the boundary film generation of various phosphonium ILs on a series of native films on metal and ceramic surfaces using multinuclear solid-state nuclear magnetic resonance (NMR) [10]. The results indicate that $\mathrm{SiO}_{2}$ and $\mathrm{Mg}(\mathrm{OH})_{2}$ interact strongly with ILs anions and cations, firstly through the adsorption of anions and subsequently by the close proximity of cations in the form of double layers (as observed through ${ }^{1} \mathrm{H}-{ }^{29} \mathrm{Si}$ cross-polarization experiments). In contrast, $\mathrm{Al}_{2} \mathrm{O}_{3}, \mathrm{MgO}, \mathrm{ZnO}$, and $\mathrm{ZrO}_{2}$ appear less active owing to less hydroxyl groups on these surfaces. Kajdas developed the anion adsorption model, in which low energy electrons are emitted from contact convex points on the metal surface during friction, leaving a positive charge formed at the surface of the tiny convex volumes, which strengthens the adsorption mode [11]. Clearly, the anions in ILs can be easily adsorbed onto these positively charged sites of a worn metallic surface, and the counter cations are assembled successively by the electroneutrality principle. The dipolar ion structure makes ILs to form an ordered bilayer crystal structure in solid state, in which alkyl chains interdigitated and an extended bilayer with alkyl chains are packed end-to-end. Therefore, it might be true that ILs form ordered adsorption layers on sliding surfaces, most possibly in multilayered fashion, whose structure resembles that of graphite and molybdenum disulfide [7, 12]. This provides good lubricity for ILs under relatively low shear strength. There are thousands of different types of ILs owing to the variable combination of cations and anions. To screen those in which the industry could be interested and that can be used in practice will be a continuous task of future research.

Because 1-hexyl-3-methylimidazolium tris(pentafluoroethyl)trifluorophosphate ([HMIm]FAP) ionic liquid is composed solely of cations and anions, the interface lubricity can be externally controlled in situ by application of a potential bias to a highly oriented pyrolytic graphite (HOPG) surface. Atkin et al. revealed that superlubricity can be "switched" on and off in situ when an ionic liquid is used to lubricate the silica-graphite interface by atomic force microscopy measurements [13]. When the potential is changed, the composition of the interfacial layer responds, which alters the frequency and magnitude of stick slip events, and hence the energy dissipated and friction. At $+1.5 \mathrm{~V}$, the anion-rich interfacial layer is "super-lubricating" and friction falls to immeasurable values.

\subsection{Friction modifier (FM) function owing to interfacial assembly}

Another very frequently used way to enhance the lubricity and antiwear of lubricants is the application of FMs (additives) [14]. FMs have polar heads that can interact with the frictional surface. Meanwhile, Van der Waals forces between the molecular tails cause the molecules to align themselves in such a way that they form the absorbed monolayer. If the timescale allows it, all molecules line up, straight, perpendicular to the metal surface, leading to a multilayer formation of the FM molecules (Fig. 4(a)). The FM layers are difficult to compress but very easy to shear at the hydrocarbon tail interfaces, which supports the loadcarrying capacity and friction-reducing properties of the FMs, respectively. FMs are usually employed in rather low concentration, but they can significantly enhance the tribological properties of base oils, especially the less polar oils.

Fatty acids have long been used as FMs to reduce friction in mineral oils owing to their high affinity to metal surfaces [15]. Therefore, it is easy to understand that their friction-reduction ability depends on the (a)

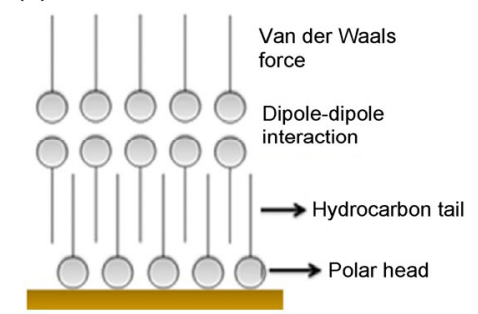

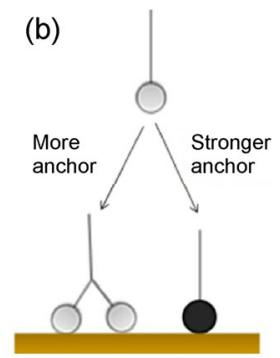

Fig. 4 (a) Interaction of friction reduction additive with frictional surface. (b) Designing novel additives. 
type of substrate, because of the differential reactivity of carboxylate with substrates. Besides, their effects will be weakened in polar synthetic fluids such as polyethers and synthetic esters because of the competitive interaction strength. One design criterion is to introduce an additional carboxyl group into the fatty acid to enable divalent interaction, so as to enhance the tribological property (Fig. 4(b)) [16]. Another design approach is to find alternative acting functional groups to replace the carboxylic head (Fig. 4(b)). Fatty acids made a great contribution to lubrication in the early years, since the 1920s, but since the 1950s were gradually overtaken by sulphur/phosphorous containing compounds, which provided greater adsorption strength with a larger variety of metallic materials. Since then, countless studies have been reported in the area. It was many years later, after their application in tribology, that the term "self-assembly" was created (usually, self-assembled monolayers (SAMs)) to elucidate the underlying science [17]. It was revealed that thiols and sulphides could form high quality monolayers on noble metal surfaces, much stronger and faster than fatty acids do on very limited oxides, such as $\mathrm{Fe}_{2} \mathrm{O}_{3}$ and $\mathrm{Al}_{2} \mathrm{O}_{3}$, which is the main reason why sulphurcontaining additives became popular after fatty acids. A fast and high-quality assembly of the additive on a frictional surface is favorable to heal the surface layer damage under severe sliding conditions.

SAMs have been one of the most important research areas in the past thirty years in surface/material science. The vast amount of research on SAMs should help tribologists to understand the underlying science more clearly, and eventually contribute to a more effective design of new lubricants. Because different head groups have different reactivity with substrate materials, there will be no universal FMs for all materials. Is there any hope to find a modifier that can adhere to all materials? The recently studied mussel-inspired adhesion, based on catecholic chemistry, provides one possibility [18]. However, the selfassembly rate of dopamine at the interface might not match with the frequency of friction. The effective FM might be that in which instantaneous assembly occurs once the absorption layers are sheared off and the assemblies are strong enough to withstand load. Besides additive design, some novel characterization approaches for SAMs should be more extensively used to understand the lubrication mechanisms at molecular level. Work on these aspects will require additional efforts.

Here we would like to mention a novel way of applying FMs by using thermo-reversible (TR) gel lubricants, which consist of a base fluid and gel agent bearing polar groups and alkyl groups [19-24]. TR gel lube includes a high percentage of gelling agent, which is basically an FM that is released when the gel changes to liquid state above the melting point. Thus, they can be easily and strongly absorbed on a metal surface with their own polar group to form a stable film. This absorbed film plays a role as an oiliness agent, and it significantly decreases friction and wear. Substantial work is still required to clarify in detail the mechanism of the TR gel lube and its tribological properties. We also report on a gel lubricant made of ionic liquids (Fig. 5) [25, 26]. The benzotriazolefunctionalized imidazolium salt can form a fibrous ordered structure in conventional ionic liquids, and it has been used as lubricant by in-situ liquefying. The obtained ionic gels have good anticorrosion ability, conductivity, and thixotropic character, which make them potential anticorrosive solid electrolytes and high performance semisolid lubricants. Especially for some electrical contacts, this ILs gel can be used at low temperatures, and it liquefies upon mechanical shearing with low friction and good antiwear properties. This application prompted us to develop a gel lubrication system by designing low molecular weight gelators (LMWGs) to gelate various lubricant oil [21-24]. We have designed and prepared a class of LMWGs, which can form random three-dimensional networks and cavities, in which liquid lubricants (such as water, mineral oil, and synthetic lubricating oil) are trapped through H-bonding, $\pi-\pi$ stacking, hydrophobic interaction, van der Waals force, London dispersion forces, and electrostatic interaction. The
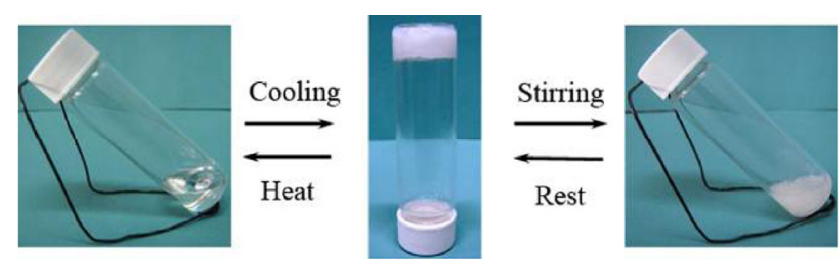

Fig. 5 A gel lubricant that reversibly changes its physical states. Reproduced with permission from Ref. [25]. Copyright Royal Society of Chemistry, 2011. 
as-prepared gels have good thermoreversible and thixotropic characteristics. This makes that gel lubricants have important implications in lubrication, in which solid-like lubricants are liquefied under high shear strength (high load and lateral shear) and high temperature, and take part in lubrication like oils. The experimental results show that these gel lubricants not only can effectively avoid the lubrication-oil creeping and evaporating loss, which benefits maintenance, but also have better lubricating and antiwear performances than the blank base lubricating liquid and grease. For some special contacts, the gelated base oil can be used to solve problems of base oil creeping and evaporating loss, and thus to enhance maintenance and operation.

\subsection{Improved aqueous lubrication by amphiphilic surfactant adsorption}

Different from the wide application of oil lubrication in industry, aqueous lubrication is used in relatively limited areas, such as metal working processes, bearings in marine industries, and biological systems. Water has limited boundary lubricating capability (load carrying capacity) owing to its low viscosity and viscous pressure coefficient. One main task of aqueous lubrication is to improve the viscosity of aqueous liquids. For example, it has been recently reported that a large-molecular-weight polyanion, sodium poly(7-oxanorbornene-2-carboxylate), can be a high performance synthetic water lubricant for biolubrication because it can significantly improve the rheological property of water [27].

Aside from the viscosity concerns, friction in aqueous media can be similarly reduced through the adsorption of amphiphilic surfactants onto substrates. Briscoe et al. found that the frictional stress between sliding surfaces coated with amphiphilic surfactant layers (N,N-dimethyl-N,N-diundecyl ammonium bromide) immersed in water could be reduced by $1-2$ orders of magnitude or more, relative to its value in dry air, although the adhesion hysteresis is comparable in both cases [28]. The surfactant head groups at the substrate surface become hydrated under water and as a result, the slip plane between the sliding surfaces shifts from the alkyl tails/alkyl tails interface in air or oil environment, to the head group/substrate interface in water. The high fluidity of the hydration sheath around the head groups results in greatly reduced friction. Sliding is much easier as a result of the hydration-lubrication mechanism of the hydrated head groups.

Lipids are another type of amphiphilic molecules in biological environments and exist mostly in form of liposomes. Goldberg et al. coated mica with small (approx. diameter $70 \mathrm{~nm}$ ) unilamellar (single bilayer wall) vesicles of hydrogenated soy phosphatidyl choline (HSPC, structure shown in Figs. 6(a)-6(c)), and investigated the friction acting between two such liposome-coated surfaces by surface force balance (SFB) [29]. The lipsomes may form close-packed boundary layers on surfaces under water, which could lead to a striking reduction in the sliding friction, with the friction coefficients down to $2 \times 10^{-5}$ at pressures up to more than $100 \mathrm{~atm}$ (Fig. 6(d)). This is attributed to hydration-lubrication by the highly-hydrated phosphocholine groups, which exposed at the surface of the liposomes rub against each other, together with the very robust nature of the adsorbed layers of vesicles. This is due to the closed structure, uniformity, and close packing of the liposomes on the surface, and particularly their rigidity by virtue of being in the gel phase, which enhances their load-carrying capacity. The extreme boundary lubricating properties of liposomes arising from the hydration lubrication mechanism thus offers great promise for medical and biomedical applications where friction is an issue, including osteoarthritis, contact lenses, or knee- or hip-joint implants [30].

\subsection{Improved aqueous lubrication by brush-like macromolecules}

Polymers cannot only increase viscosity, but have potential to form boundary adsorption, and thus are an important choice for water lubricants. They are largely inspired by lubrication in nature. The friction coefficient of articular cartilage can be as low as 0.001 , and it takes advantage of water lubrication through brush-like glycoproteins grafted on the surface or in bulk, which retain water molecules to generate repulsive hydration forces at the interface of the sliding surfaces (Figs. 7(a) and 7(b)) [31-33]. The presence of hydrated glycoprotein at the friction surface simply increases the interface viscosity of water and makes it 


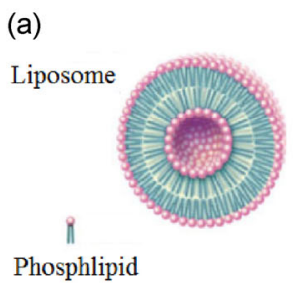

(b)

(c)

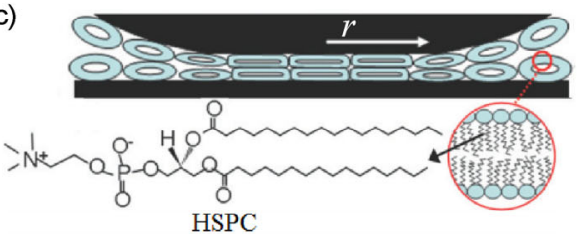

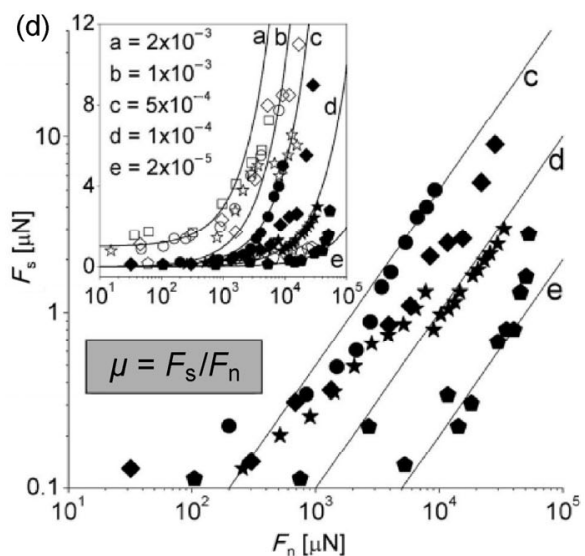

Fig. 6 (a) Liposome, (b) cryo-SEM image of the HSPC-SUV adsorbed on freshly cleaved mica, (c) scheme of HSPC-SUV self-assemble in close-packed layers on mica surfaces, and (d) friction forces $F_{\mathrm{s}}$ versus applied loads $F_{\mathrm{n}}$ between two HSPC-SUV-coated mica surfaces (as in Fig. 6(c)). The inset shows the $F_{\mathrm{s}}$ versus $F_{\mathrm{n}}$ variation on a first approach (empty symbols), and on second or subsequent approaches (correspondingly filed symbols). Curves a-e show the variation $F_{\mathrm{s}}=\mu F_{\mathrm{n}}$, with friction coefficient $\mu$ values as indicated in the inset [29, 30]. Reproduced with permission from Ref. [29]. Copyright WILEY-VCH Verlag GmbH \& Co. KGaA, Weinheim, 2011.

(a)

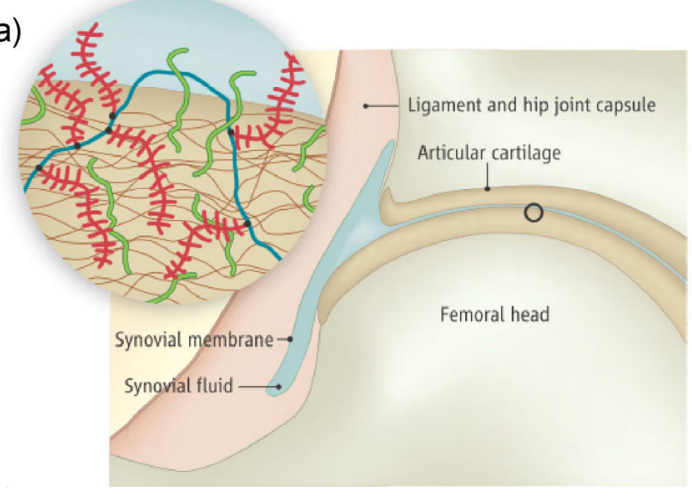

(b)

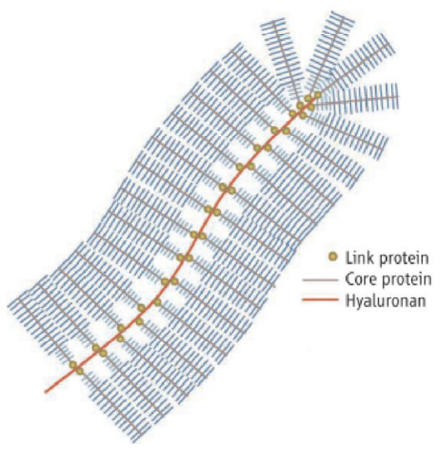

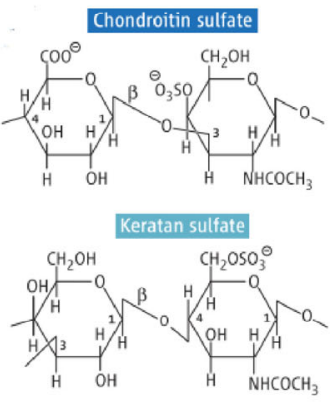

Fig. 7 (a) The close-up view of the articular cartilage surface. (Inset) The detailed structure at the outer cartilage surface is thought to include charged macro-molecules, mainly hyaluronic acid (blue), to which are attached aggrecans (red) and lubricins (green) that extend from the surface to form a brush-like layer. Reproduced with permission from Ref. [33]. Copyright American Association for the Advancement of Science, 2009. (b) Illustration of the bottle-brushes structure of aggrecans. Reproduced with permission from Ref. [32]. Copyright American Association for the Advancement of Science, 2008.

a good lubricant. In a good solvent, the high polymer grafting density forces the polymer chain to stretch away from the surface to avoid overlap of the molecular chain [18]. When the counter sliding pair approaches and presses the polymer chains, the crowded polymer chains will produce a very large osmotic pressure that generates a direction opposite to the applied load through osmolality exclusion. Macromolecular brushes form a hydration layer that is easy to flow, leading to ultra-low friction.

However, whether a hydrophilic polymer can result in ultralow friction depends on its capability to form an effective boundary layer. For a rational polymer design, the polymer structures listed in Fig. 8 are highly desirable. They are either diblock polymers, or end functionalized polymers, or combing polymers with anchoring groups, wherein one compartment

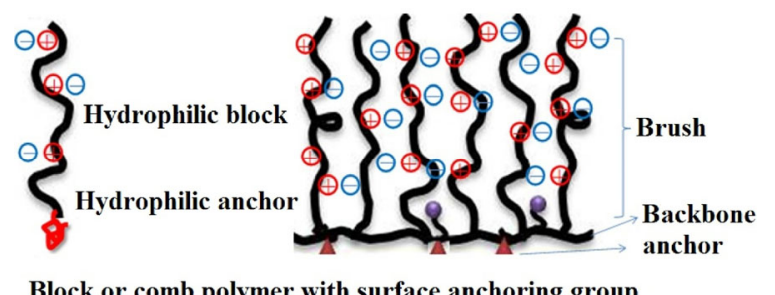

Fig. 8 Structures of hydrophilic polymers with surface anchoring groups. 
forms strong interaction with the frictional surface and the other hydrophilic part forms a hydration layer. Klein et al. reported on the water lubrication behavior of the poly(methylmethacrylate)-block-poly (sodium sulphonated glycidyl methacrylate) copolymer (PMMA-b-PSGMA) on the hydrophobic surface of mica. PMMA segments have strong hydrophobic interaction with the mica surface, which is able to anchor in-situ the anionic PSGMA onto the frictional surface, with polymer chains stretching outwards to form a "brush" type structure [31]. Figure 9 shows that the polymer surfactants lead to very low effective friction coefficients, whereas the neutral brushes and the adsorbed charged chains (coloured symbols and bands) show a rapid increase in friction coefficients at higher volume fractions. Friction mediated by PSGMA brushes (black symbols and grey band) remains extremely low, up to the point where the volume fraction is close to unity. This is attributed to the exceptional resistance to mutual interpenetration displayed by the compressed, counterion-swollen brushes, together with the fluidity of the hydration layers surrounding the charged, rubbing polymer segments. Spencer et al. studied the tribological properties of the positively charged lysine as main chain, hydrophilic polyethylene glycol as side chain, and "bottle brush" type polymer (PLL-g-PEG) as a waterlubricated additive [34,35]. The polypeptide backbone with a positive charge can be anchored to the negative

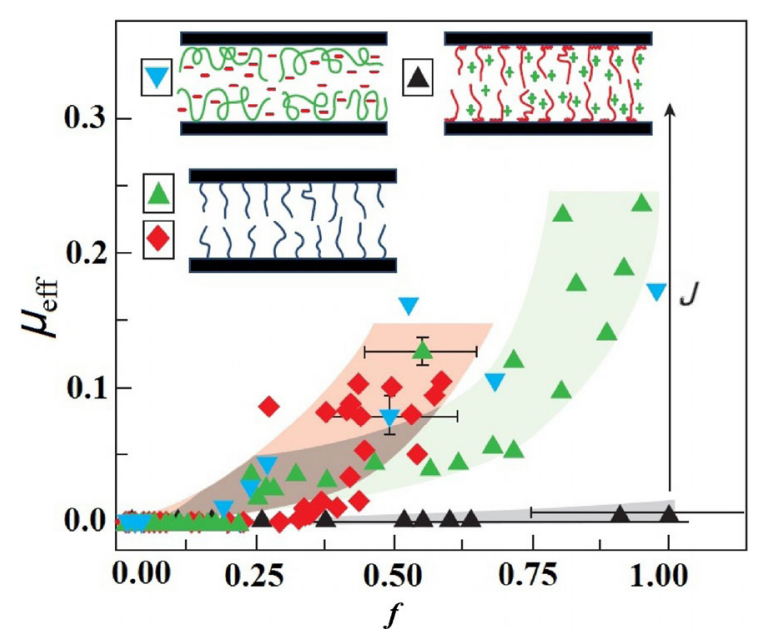

Fig. 9 Variation of the effective friction coefficient $\mu$ with volume fraction $f$ of confined polymer for different polymer lubricants. Reproduced with permission from Ref. [31]. Copyright Managed by Nature Publishing Group, 2003. charge of the metal and the oxide surface by electrostatic interaction. The PEG chain extending outwards forms a "brush"-type structure, and it is combined with a large number of water molecules, which play a very good boundary lubrication effect through an increase in the chain length of the side chain PEG segment, to obtain a lower friction coefficient. They also studied the self-healing effect of the water-soluble polymer additives in the process of friction. When the boundary lubrication layer is damaged because of wear, the bulk polymer in the solution is able to repair the wear surface quickly through electrostatic interactions, which maintains the friction coefficient low.

\subsection{Improved aqueous lubrication by surface grafted polymer brushes}

Surface-initiated polymerization from a surface bound initiator monolayer offers the easiest way to prepare surface-grafted polymer brushes in high density, and it can be used for aqueous lubrication. Spencer et al. have used a photoinduced "grafting from" approach to grow polymethacrylic acid (PMAA) brushes on $\mathrm{Si} / \mathrm{SiO}_{2}$ surfaces, and investigated the lubrication properties of the PMAA brushes of different molecular weights and soft, hydrophilic ox-PDMS pins in macroscopic tribological experiments under lowcontact-pressure, aqueous conditions [36]. The results showed that the PMAA brushes could not be distinguished from the $\mu$ vs. sliding speed plots, and long-term stability of the short $15 \mathrm{~nm}$ PMAA brushes showed to be inferior to that of long brushes $(240 \mathrm{~nm}$ dry thickness). PMAA brushes display significantly lower friction than PEG monolayers under aqueous lubrication conditions. This is mainly due to the higher grafting densities of the PMAA compared to PEG layers, enhanced swelling of the polyelectrolyte brushes in neutral aqueous media, and additional electrostatic repulsion against the oxidized PDMS slider. Klein et al. used a surface force apparatus to study the behavior of water-lubricated mica surfaces with grafted polymer PMPC brushes, and found that the surfaces can have $\mu$ values as low as 0.0004 at pressures as high as $7.5 \mathrm{MPa}$ (Fig. 10) [37]. The primary mechanism underlying the low friction was attributed to the side-chain phosphorylcholine group, capable of binding a large number of water molecules and 


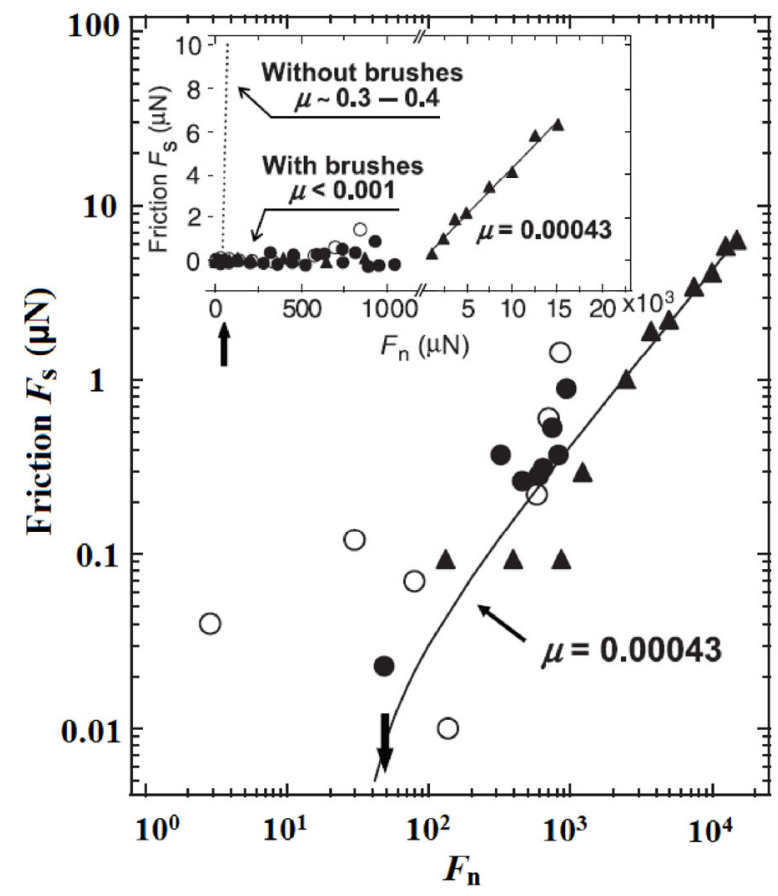

Fig. 10 Variation of friction $F_{\mathrm{s}}$ with load $F_{\mathrm{n}}$ and with sliding velocity, and vs. PMPC brush-coated surfaces. Reproduced with permission from Ref. [37]. Copyright American Association for the Advancement of Science, 2009.

allowing easy flow of the hydration layer. The flexibility of the hydration layer can withstand high loads and maintain a very low friction coefficient.

Friction and wear are often an issue in human-made systems. These results may have relevance for boundary lubrication in human-made systems, and they reveal potential in aqueous or physiological media, especially for biomedical devices.

The interactions between the responsive polymer brushes are strongly dependent on the conformation of the brush, which can be finely controlled. The change in conformation of polyelectrolyte brushes can be translated into friction control [38]. Rutland and Nordgren investigated the stimuli-induced lubrication behavior between poly(2-(dimethylamino)ethyl methacrylate) (PDMAEMA) grafts on gold under different $\mathrm{pH}$ and temperature environments with colloidal probe atomic force microscopy, and found out friction response to $\mathrm{pH}$ and temperature [39]. The effects of these two parameters on the brush collapse are subtly different, because $\mathrm{pH}$ affects primarily the charging of the amine moieties, whereas temperature controls the strength of the hydrophobic interactions between the polymer chains and the solvent. At low $\mathrm{pH}$, the brush with low friction was attributed to the formation of a repulsive, highly charged, hydrated cushion. At high $\mathrm{pH}$, the friction was significantly increased. The system turned attractive above the lower critical solution temperature, with a small friction reduction interpreted as being due to nanoscopic flattening at the interfacial boundary.

Despite several works being conducted on tuning friction of polyelectrolyte, the types of counterions and the magnitude of friction change are still very limited. We systematically present feasible surfaces for dramatically tuning macroscale friction from superior lubrication $\left(\mu \sim 10^{-3}\right)$ to ultrahigh friction $(\mu>1)$ via exchanging counterions into polyelectrolyte brushes when the surface is sliding against a silicone elastomer ball [38]. The tunable friction is based on counteriondriven interactions in polyelectrolyte brushes that can be simply achieved by exchanging the counterions. The effects of opposite counterions of different types on the friction properties of polyanionic, polycationic, and polyzwitterionic brushes were systematically investigated. Figure 11(b) showed that the friction coefficient of cationic brushes with quaternary ammonium groups was progressively tuned from $\sim 10^{-3}$ to $\sim 10^{0}$ according to the counterions series $\mathrm{Cl}^{-}$ $<\mathrm{ClO}_{4}{ }^{-}<\mathrm{PF}_{6}{ }^{-}<\mathrm{TFSI}^{-}$. The friction of anionic brushes can be tuned by oppositely charged surfactants (tetraalkylammonium) with different length of hydrophobic tails, multivalent metal ions, and protons. The friction increase of cationic brushes is due to the dehydration and the collapse of polyelectrolyte chains induced by ion-pairing interactions. For anionic brushes, the friction increased with the length of hydrophobic tails of surfactants, which resulted from hydrophobicityinduced electrostatic interaction among the surfactants and polymer chains (Fig. 11(c)). The anionic brushes with the carboxylate and the sulfonate side groups revealed different friction responses, which is due to the carboxylate groups getting a stronger specific interaction with the quaternary ammonium, and thus, with the multivalent metal ions. The mechanism of tuning friction was finally concluded; that is, highly hydrated and swelling polymer brushes show superior lubrication, partially collapsed polymer chains have moderate lubrication, while the completely dehydrated 
(a)
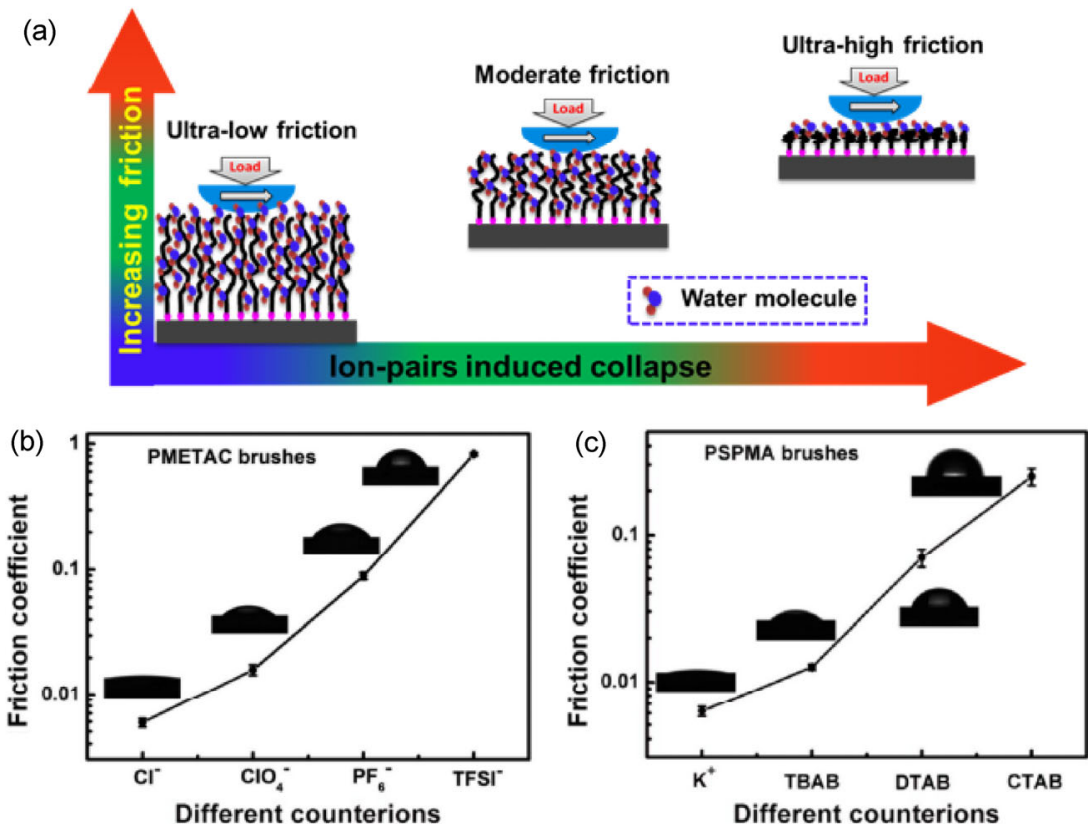

Fig. 11 (a) Illustration of correlation between lubrication and conformation of polymer brushes, changes in friction coefficients and wettability (inserted images) corresponding to (b) and (c). (b) PMETAC brush on $\mathrm{Si}$ substrates when the counterions are $\mathrm{Cl}^{-}, \mathrm{ClO}_{4}^{-}$, $\mathrm{PF}_{6}{ }^{-}$, and $\mathrm{TFSI}^{-}$. (c) Polyanionic brushes on Si substrates; the counterions are cationic surfactants (TBAB, DTAB, and CTAB) for PSPMA brushes. Reproduced with permission from Ref. [38]. Copyright American Chemical Society, 2013.

and collapsed conformation loses lubricating capability (Fig. 11(a)). The tunable lubrication platform would be helpful to further understand the lubricating mechanism of polymer brushes and would promote applications of these smart surfaces in micro fluidic devices, biosensors, and so on.

Recently, our group found that the weak interaction between polymers and surfactants played an important role in the lubrication behavior and wettability of polymer brushes, which depended on the surfactant concentration [40]. Taking cationic (PMETAC) and anionic (PSPMA) brushes as examples, with the addition of surfactant above the critical micelle concentration (CMC), the weak interaction achieved a gradual transition from ultralow friction to ultrahigh friction, because the surfactant exchange led to a strong dehydration of the brush. When the addition of surfactant was above the $\mathrm{CMC}$, a reduction in friction for the anionic brushes was observed, owing to the lubricating effect of micelles, while the cationic brushes maintain a high friction, without this micelle lubricating effect. These results reveal the relation between surfactant adsorption and lubrication behavior.

\subsection{Improved wear resistant by surface grafted polymer brushes}

Migration of the artificial femoral head to the inside of the pelvis caused by the degeneration of the acetabular cartilage has emerged as a serious issue in resurfacing or bipolar hemi-arthroplasty [41]. Surface modification of the cobalt-chromium-molybdenum alloy (Co-Cr-Mo) is one of the promising means of improving lubrication for preventing the migration of the artificial femoral head. Ishihara et al. systematically investigated the surface properties of the various surface modification layers formed on the $\mathrm{Co}-\mathrm{Cr}-\mathrm{Mo}$ surface by the MPC polymer by dip coating or photoinduced radical grafting. The results showed that the cartilage/poly(MPC) (PMPC)-grafted Co-Cr-Mo interface mimicked a natural joint, with an extremely low friction coefficient of $<0.01$, as low as that of a natural cartilage interface. Moreover, in the PMPCgrafted layer no hydrolysis of the siloxane bond was observed throughout soaking in phosphate-buffered saline for 12 weeks, and it showed a long-term stability in water. These results illustrated that 
cartilage/poly(MPC) (PMPC)-grafted Co-Cr-Mo interface prevented degeneration of the articular cartilage. Current artificial joints with polyethylene (PE) surfaces have considerably less efficient lubrication and thus much greater wear, leading to osteolysis and aseptic loosening. Then, they reported the development of nanometer-scale hydrophilic layers with varying charge (nonionic, cationic, anionic, or zwitterionic) on cross-linked PE (CLPE) surfaces, which could fully mimic the hydrophilicity and lubricity of the natural joint surface [42]. The POEGMA- and PMPC-grafted CLPE cups exhibited high wear resistance in the hip simulator tests along with low coefficients of dynamic friction in the ball-on-plate friction tests. The primary mechanism underlying the low friction and high wear resistance must be attributed to the high level of hydration of the grafted layer, where water molecules act as very efficient lubricants. The secondary mechanism is repulsion of protein molecules and positively charged inorganic ions by the grafted polyelectrolyte layer.

Many excellent theoretical and computer simulation studies have been performed to elucidate the mechanism responsible for low friction between polymer brushes. However, to extend these insights to sliding layers is a challenge. Goujon et al. have calculated the kinetic friction coefficient of neutral and charged polymer brushes with the same grafting density at different compression ratios, in an effort to model SFB experiments in these systems using dissipative particle dynamics (DPD). They found that the kinetic friction coefficient between the charged brushes is higher than that of neutral brushes at low compressions and smaller at high compressions, although the differences remain small. They also showed that it is possible to simulate ultra-low friction coefficients, comparable with experiments, using the smallest shear rate accessible in a mesoscale simulation. The polyelectrolyte brushes can support a higher load due to the presence of free counterions within the brush. The viscosity in the polymer free gap is smaller in charged brushes than in neutral brushes, indicating that the solvent particles in this region assist the sliding of the two interacting charged brushes. The unexpected behavior of the shear deformation-induced structural heterogeneities in charged polymer brushes calls for further experiments to elucidate this local reorganization of the ions in adsorbed charged polymers.

The surface graft polymer brushes have a low coefficient of friction in a water environment, but are usually worn out within 50-100 cycles of macroscopic reciprocating friction by the sliding probe under a high normal load of around $100 \mathrm{MPa}$, resulting in a high friction coefficient owing to the exposure of the bare substrate. Thus, the wear resistance for the application of a polymer brush is not sufficient for its practical use under load bearing conditions. However, it is not difficult to solve this issue, because the polymer brushes composition and structure can be flexibly designed by researchers. Takahara et al. reported that partially cross-linked ion-containing polymer brushes were effective for the improvement of wear resistance without loss of good lubrication. They prepared copolymer brushes of 2-dimethylaminoethyl methacrylate (DMAEMA), 2-(methacryloyloxy)ethyl phosphorylcholine (MPC), 2-(methacryloyloxy)ethyl trimethylammonium chloride) (METAC), and 3-sulfopropyl methacrylate potassium salt (SPMK) with excellent waterwettability [43]. Among them, the poly(SPMK) revealed an extremely low friction coefficient of around 0.01, even after 450 friction cycles. Furthermore, the poly(SPMK-co-METAC) brush with electrostatically cross-linked structure showed an extremely low friction coefficient in water, even after 1,400 friction cycles under a pressure of $139 \mathrm{MPa}$. The cross-linking structure improved the shear strength of the polymer brush thin films, thus preventing wear of the brush, and maintaining hydrophilicity to assist water lubrication.

From the above, lowering friction can be basically achieved by interface adsorption of small molecules in oil lubrication, so that viscous shearing can be transformed to shearing between an ordered molecular assembly or by adsorption of hydrated molecules to generate hydration lubrication in water environment. A comparison of research on aqueous and oil lubrication would be of benefit to both. One question is whether it is possible to achieve ultralow friction in an oil lubricating system, and how. How can stable ultralow friction be achieved under most engineering conditions, e.g., high loads, engineering surfaces rather than atomic flat surfaces, and conformal surfaces? 


\section{Friction reduction and antiwear based on tribochemistry}

In mixed and boundary lubrication, surface asperities come to contact, deform, and break down, as seen in Fig. 12(a). Complex tribophysicochemical processes occur, which include local temperature increase, wear of local asperity to generate a fresh nascent surface, and triboemission (electrons, ions, photons, etc.). Boundary adsorption films can prevent direct contact of asperities (Fig. 12(b)). Flash temperature is generated at contacted asperities, and it is usually short lived. If the heat cannot be dissipated in time, it would lead to increased temperature that exposes lubricant molecules to thermal stress and speeds up a tribochemical reaction. As shown in Fig. 12(c), when asperities are worn off, a nascent surface is generated with dangling bonds, and it is far more reactive than the native surface to activated reactions [44]. Another tribophysical process is the triboemission, the emission of electrons, ions, photons, UV radiation, IR radiation, and acoustic emission, as a response to friction and wear processes [45]. The spontaneous emission of low-energy electrons (exoelectrons) from solid surfaces comes from plastic deformation, abrasion, fatigue cracking, or phase transformation. The positive charges or electron vacancies left on the frictional surface, though having only a minute life time, are sufficient to initiate chemical reactions with molecules of the environment. Triboplasma (Fig. 13) may be generated by discharges in the surrounding gas owing to triboelectrification [46-48]. In the vicinity of the sliding contact reactions, it can cause tribochemical reactions $[47,49]$.

\subsection{Tribochemical reactions}

Tribochemistry generally refers to the chemical reactions that occur between the lubricant and the rubbing surfaces under boundary lubrication conditions [51]. It may have harmful effects by accelerating the decomposition of lubricants or have beneficial effects by forming friction and wear reduction organic films. The protective films are usually formed at much lower temperatures than those at which lubricants normally show chemical activity, indicating a catalytic reaction by friction. Tribochemistry is a complex process, which is very difficult to fully understand, and could not always be explained with the traditional static reaction mechanisms of thermal and catalytic reactions, but also must be governed by those chemical reactions under the dynamic triboprocess. For example, zinc dialkyl dithiophosphates (ZDDPs), as very effective comprehensive additive in versatile base oils, have been used for dozens of years. However, the underlying mechanisms have not been fully disclosed so far, and thus, it is still a key topic. Beyond surface adsorption, tribochemical reaction is the main functioning way of friction reduction and antiwear additives.
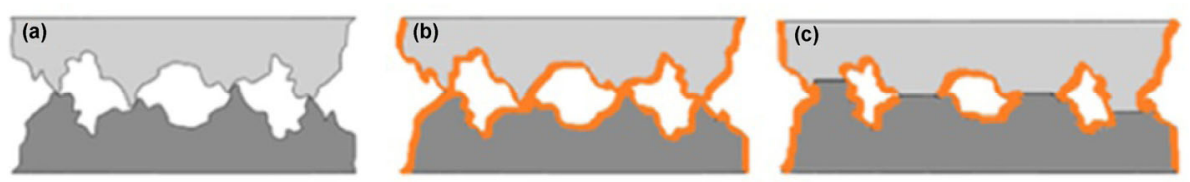

Fig. 12 The contact complications in mixed and boundary lubrication

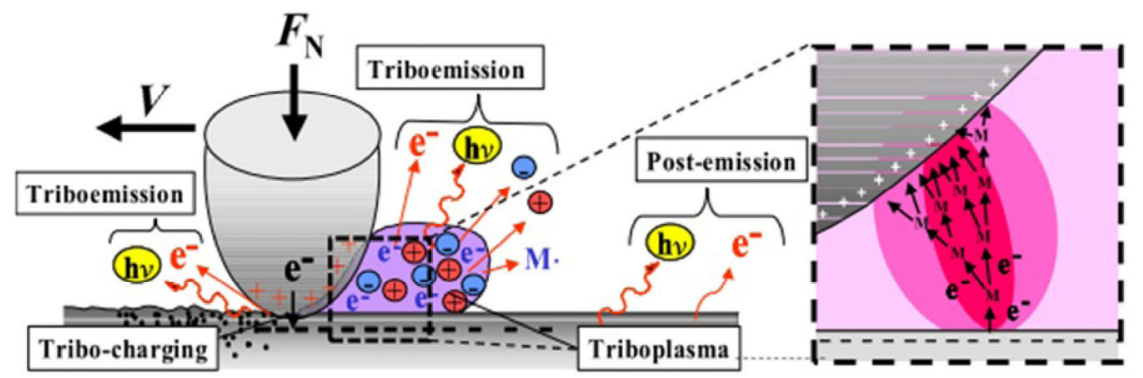

Fig. 13 Model of triboplasma generation through discharging of gas owing to triboelectrification [46, 47, 50]. Reproduced with permission from Ref. [46]. Copyright Elsevier, 2004. Reproduced with permission from Ref. [50]. Copyright Elsevier, 2006. 


\subsection{DDPs based additives}

ZDDP reacts with asperities to reduce the contact and when the load is high enough to collapse the oil film, it reacts with the entire metal surface to prevent welding and to reduce wear [51]. Most of the attention in recent years has been focused on the ability of ZDDP to form tribofilms on rubbing iron and steel surfaces under mild wear conditions and, in particular, on the chemical composition and properties of these films. They consist primarily of closely packed pads of glassy, zinc or tho- and pyrophosphate, typically $5 \mu \mathrm{m}$ across and $100 \mathrm{~nm}$ thick [52]. The outermost layers of these pads contain a longer chain zinc polyphosphate. The lower layers next to the surface contain some iron cations in place of zinc. Between the pads there are fissures containing a bulk solution. Under more severe conditions, they also act as an extreme pressure additive to form metal sulphides and the phosphate glass to prevent cold welding. Mosey carried out a computer simulation of zinc dialkyl dithiophosphates under extreme conditions and concluded that pressure-induced cross-linking is a key mechanism in the formation and functionality of antiwear films [53]. Recently, Gosvami et al. monitored in-situ the growth and properties of the tribofilms in well-defined single-asperity sliding nanocontacts [54]. They found that the growth of ZDDP antiwear tribofilm increases exponentially with the applied pressure and temperature. This result is consistent with the stress-assisted reaction rate theory. The research provides a new way to study tribochemical phenomena and functionality at the molecular scale.

Following the application of ZDDPs, many analogue compounds have been developed. Among these compounds, molybdenum dithiophosphates (MoDTP) and molybdenum dithiocarbamate (MoDTC) have been extensively studied. Grossiord et al. have investigated the mechanisms of MoDTC for reducing friction under boundary lubrication using analytical tribometry [56]. The C-based tribofilm contains a few percent of highly dispersed $\mathrm{MoS}_{2}$ in the form of individual sheets of length less than $10 \mathrm{~nm}$. A mechanism of the breakdown of the MoDTC molecule from a chemical point of view was proposed, as shown in Fig. 14 [55, 56]. The initial step towards $\mathrm{MoS}_{2}$ formation is via electron (a)

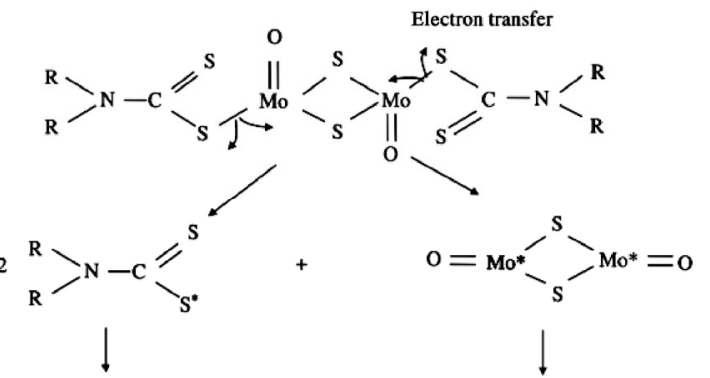

(c)

(b)
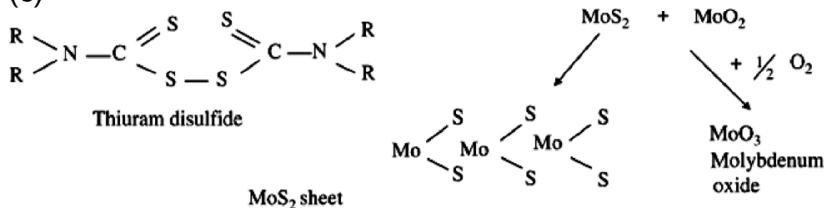

Fig. 14 Molybdenum dialkyl dithiocarbamate (MoDTC) decomposition chemical model $[55,56]$. Reproduced with permission from Ref. [56]. Copyright Elsevier, 1999.

transfer at the Mo-S chemical bonding MoDTC (Fig. 14(a)), leading to the formation of three free radicals (Fig. 14(b)): two at the core of MoDTC and the other one at the chain end. The third step is core radical decomposition (Fig. 14(c)) into $\mathrm{MoS}_{2}$ and $\mathrm{MoO}_{2}$ which can be further oxidised, and the recombination of chain end radicals forming thiuram disulphides. The layer-lattice structure of the molybdenum disulphide makes it possible to achieve low friction. The thiuram disulphides form the interlayer, having weak van der Waals force, and is easy to shear resulting in a low coefficient of friction. Besides, the organic molybdenum additives (DDP based and DTC based) contain several polar atoms such as $\mathrm{S}, \mathrm{P}$, and $\mathrm{N}$. These strong activity components can react with the substrate materials, such as steel, to form boundary friction reduction and antiwear films consisting of $\mathrm{MoS}_{2}, \mathrm{FePO}_{4}, \mathrm{FeS}$, and $\mathrm{FeO}$, which can prevent the substrate from cold welding. The effectiveness of MoDTC in reducing friction is strongly affected by synergistic or antagonistic effects with other additives $[52,53]$, especially ZDDP. When ZDDP is used with MoDTC, it helps to produce $\mathrm{MoS}_{2}$ and benefits the lubrication performance.

With the growth in awareness of the environmental crisis, the pollution problem from lubricating oils is receiving increased attention. The possible solutions are to use environmentally friendly lubricants and additives. Therefore, there has been an increasing demand for developing environmentally friendly 
lubricants and additives with low-sulfur and lowphosphorus have been of great interest in tribochemical research in the past decade. Novel zinc-free compounds, alkyl borate-dithiophosphates, with a low content of sulphur and phosphorus, are designed as additives for lubricants [57]. Their interfacial performance in steel-to-steel contacts in a mineral oil was studied in terms of friction and antiwear characteristics. This class combines two high-iron-affinity surface active groups, borate and dialkyl dithiophosphate, into a single molecule. The results show that alkyl boratedithiophosphates, with substantially reduced amounts of sulphur and phosphorus compared with $\mathrm{Zn}-\mathrm{Bu}$ DTP, have considerably better antiwear and friction performance. This is mainly because tribofilms, formed on the steel surfaces when the novel additives are admixed in the mineral oil, enhance steel-to-steel contact performance. Metal-, sulphur-, and phosphorous-free additives will be a long-term task.

\subsection{Ionic liquids additives}

For the above mentioned target, ILs represent a good choice to be considered. As mentioned before, ionic liquids, as the base oils, exhibit excellent tribological behavior from fluid lubrication to boundary lubrication $[4,58,59]$. Except for the polarity-induced surface absorption, under harsh sliding conditions, the active elements $[N, F, P(B)]$ in ionic liquids offer sufficiently rich tribochemistry to form extreme pressure films of lower shearing strength by reacting with the fresh surface, which leads to lower friction coefficient and good wear resistance. It was found that ionic liquids as the additives in base oils also perform very well [60-75]. The tribochemical reactions of ILs have been extensively studied. Qu et al. [9] have characterized the boundary lubricating film formation of an ionic liquid (IL) trihexyltetradecylphosphoniumbis(2ethylhexyl) phosphate as potential antiwear lubricant additive in polyalphaolefin oil. Figure 15(a) shows a cross-section of the near surface region underneath the wear scar lubricated by PAO+IL(5\%). It clearly shows a two-layer structure including a top triboboundary film (120-180 nm) and a subsurface plastic deformation zone $(0.5-0.8 \mu \mathrm{m})$ with a refined grain structure. This protective boundary film is responsible for the antiscuffing/antiwear functionality. The higher-magnification image of the boundary film in

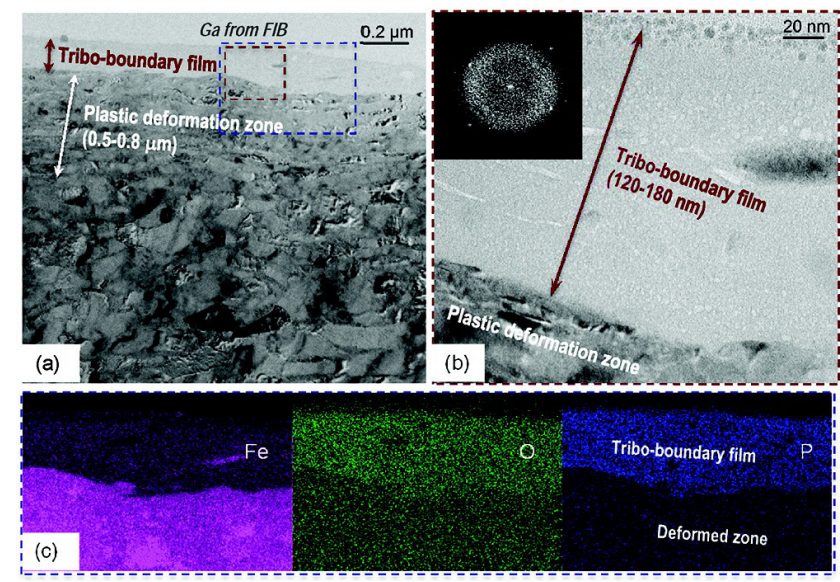

Fig. 15 TEM imaging and occurred EDS element mapping on the cross-section of the near surface zone of a cast iron worn surface lubricated by PAO+IL(5\%). (b) and (c) correspond to the red dashed line box and blue dotted line box marked on (a), respectively. Reproduced with permission from Ref. [9]. Copyright American Chemical Society, 2012.

Fig. 15(b) (corresponding to the red dashed box in Fig. 15(a)) reveals an amorphous matrix embedded with fine nanoparticles (1-10 $\mathrm{nm}$ in diameter), and the electron diffraction pattern (insert in Fig. 15(b)) confirms the nanocomposite phase structure. The EDS element mapping in Fig. 15(c) (corresponding to the blue dashed box in Fig. 15(a)) shows high concentrations of $\mathrm{P}, \mathrm{O}$, and $\mathrm{Fe}$ in the boundary film, because of interactions/reactions between the IL and the metal surface.

Despite of their exceptional tribological property, the thermo-oxidative stability is a more useful parameter of ILs for practical applications. When the imidazoliumbased and ammonium-based ILs are kept at $200{ }^{\circ} \mathrm{C}$ for $1,000 \mathrm{~h}$, the color of the samples will turn to black after long time thermal stress, probably owing to the Hofmann elimination reactions [76, 77]. Corrosion of ILs is primarily the result of the hydrolysis of perfluoro-anions such as $\mathrm{BF}_{4}^{-}$and $\mathrm{PF}_{6}^{-}$, or from impurities like halogens introduced during synthesis $[58,78]$. Thus, thermal oxidization and corrosion are two problems of ILs lubricants in real applications. An effective way to enhance the anticorrosive and antioxidative properties of ILs is to introduce functional groups. As shown in Figs. 16(a)-16(c), by incorporating benzotriazole and sterically hindered phenol, or both, into imidazolium, the anticorrosion and antioxidation properties could be considerably enhanced when 
these compounds were used as the anti-wear (AW) additives in polyether [79-81]. The molecular design is to introduce more tribochemical reactions that can inhibit oxidation and corrosion for better protection of base oils. It has been recently found that ionic liquids could be formed in base liquid in-situ by blending TFSILi with polyether and polyol ester [82-85]. Lithium bis(trifluoromethylsulfonyl)imide (LiTFSI) can form ILs with synthetic esters, [Li(synthetic ester)]TFSI, by the donation of lone pairs on carbonyl oxygen atoms of an ester molecule to a lithium ion to form a weakly Lewis-acidic complex cation [Li(synthetic ester) $]^{+}$and the following interaction with the weakly Lewisbasic anion TFSI $^{-}$to generate [Li(synthetic ester)]TFSI. LiTFSI interacts with polyether in a very similar way. The easy preparation, extremely good solubility, and excellent tribological properties of the in-situ formed ILs provide them with great advantages when compared with conventional ILs, which can hardly be used as lubricant additives in synthetic esters owing to their low solubility (Fig. 16(d)).

Both DDP type of additives, ILs, and many others have multiple active elements in molecules, which allows a rich tribochemical reaction to occur during friction. The tribochemical reaction products prevent direct contact between the sliding pairs, which is the basis of friction reduction and antiwear. The $\mathrm{S}, \mathrm{P}, \mathrm{N}$, and $\mathrm{F}$ elements are mostly seen in these additives. There are strong demands to reduce their content owing to the increased environmental pressure. Therefore, one critical topic is whether we can find alternatives that are environmentally friendly, while maintaining enough reaction activities to form boundary protecting films through triboreaction. Potential solutions include (1) designing $\mathrm{B}, \mathrm{N}$ based additives;
(2) developing inorganic additives, as will be reviewed later; and (3) developing oiliness lubrication techniques.

\subsection{Superlubricity owing to tribochemistry}

The state in which friction between two solids is zero and the solids slide without resistance to motion is called "superlubricity" [87]. Because of the limits in measurement precision, in practical terms, the lubrication state can be considered as superlubricity when the friction coefficient is lower than 0.01 in a sliding system [1]. At present, superlubricity materials include solid lubricants (diamond like carbon (DLC), molybdenum disulfide $\left(\mathrm{MoS}_{2}\right)$, etc.) [88-90] and liquid lubricants [91-97]. The solid lubricants have been studied in detail by molecular dynamics simulations and experimental investigations. However, the investigation on liquid lubricants for superlubricity is still at the early stage. Li et al. found the novel superlubricity phenomenon of phosphoric acid $\left(\mathrm{H}_{3} \mathrm{PO}_{4}\right)$ under ambient conditions [91,98]. An ultralow friction coefficient of about 0.004 between glass $/ \mathrm{Si}_{3} \mathrm{~N}_{4}$ and sapphire/sapphire tribopairs was obtained under the lubrication of a phosphoric acid aqueous solution ( $\mathrm{pH}$ 1.5) at high contact pressure (the maximum pressure can reach about $1.65 \mathrm{GPa}$ ), after a running-in period of about $600 \mathrm{~s}$. The tribological results showed that the whole friction process could be divided into three stages according to the evolution of friction coefficient with time. The first stage, from $0 \mathrm{~s}$ to $160 \mathrm{~s}$, corresponded to the process of friction rapidly decreasing. In this first stage, the mechanical action between the two surfaces plays the dominant role instead of the chemical reaction. A severe wear occurred on the friction surfaces, and as a result, the contact area increased rapidly and the contact pressure was
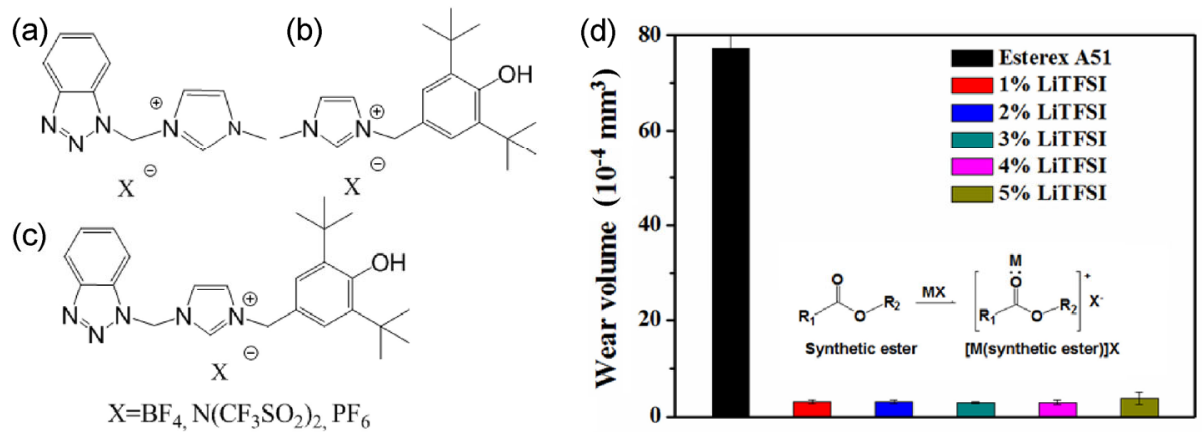

Fig. 16 Functionalized ionic liquids [79-81], and in-situ formed ionic liquids as the additive [86]. Reproduced with permission from Ref. [86]. Copyright American Chemical Society, 2012. 
reduced consequently. In addition, the tribochemical reaction between hydrogen ions and friction surfaces occurred in the contact region $\left(\mathrm{SiOH}+\mathrm{H}^{+}=\mathrm{SiOH}_{2}{ }^{+}\right)$ during the first stage, which would make the surfaces to become positively charged. The surface charge can produce the stern layer, and the electrical double layer reduces their friction coefficient. The second stage, from $160 \mathrm{~s}$ to $520 \mathrm{~s}$, was a slowly friction-reducing process. In this stage, no wear is occurring but the volume of solution is reduced to a limited amount to form the starvation state, and a hydrogen bond network among $\left(\mathrm{H}_{3} \mathrm{PO}_{4}, \mathrm{H}_{2} \mathrm{PO}_{4}{ }^{+}\right.$, and $\left.\mathrm{H}_{2} \mathrm{O}\right)$ is formed in the confined solution. The third stage, from $520 \mathrm{~s}$ to the end of the test, was the superlubricity process. In this stage, the superlubricity would keep constant if a thin film with hydrogen bond network among $\mathrm{H}_{3} \mathrm{PO}_{4}, \mathrm{H}_{2} \mathrm{PO}_{4}{ }^{+}$, and $\mathrm{H}_{2} \mathrm{O}$ is adsorbed on the track to bear the load. Therefore, the superlubricity mechanism of phosphoric acid is attributed to the hydrogen bond network between the phosphoric acid and water molecules formed on the stern layer that is induced by the attached hydrogen ions [91, 92, 98, 99].

Except the experimental investigations, reactive molecular dynamics simulations are conducted to investigate the superlubricity mechanism induced by tribochemical reactions in the silica/phosphoric acid system [100]. The friction coefficient is found to have strong positive correlation with the number of interfacial hydrogen bonds, which suggests that a weaker interfacial hydrogen bond network would favor a lower friction. The sliding friction behaviors and tribochemistry of phosphoric acid under solid confinement and shear have a strong temperature dependence in a wide temperature range. The friction reduction mechanisms have been analyzed in two temperature regimes. For $300 \leq T \leq 600 \mathrm{~K}$, no indication of tribochemical reaction is observed, and the friction coefficient decreases because of the accelerated molecular rotational and translational motion and the corresponding weakened hydrogen bond network. For $800 \mathrm{~K} \leq \mathrm{T} \leq 1,400 \mathrm{~K}$, the occurrence of tribochemical reactions leads to a clustering and polymerization of the phosphoric acid molecules and generation of a considerable quantity of water molecules, and formation of slip planes is also enhanced by the increasing temperature. It was observed the generation of water molecules and their accumulation at the sliding interface, which could lead to an even weaker interfacial hydrogen bond interaction, owing to the much faster dynamics of water molecules. Hence, it shows good lubricating properties with a friction coefficient as low as 0.02 .

In 2008, Matta et al. observed a new superlubricity macrosystem of pure glycerol [1]. They found that the use of steel surfaces and/or ta-C coated surfaces, lubricated by selected polyhydric alcohol molecules, could give unsurpassed low friction values, below 0.01 . This was attributed to the existence of a network of H-bond interactions in the confined interface zone. In addition to the formation of $\mathrm{OH}$-terminated surfaces but at a lower temperature, that super low friction of polyhydric alcohols could also be associated with the triboinduced degradation of glycerol, producing a nanometer-thick film containing organic acids and water. An outstanding superlubricity of steel surfaces directly lubricated by a solution of myo-inositol (also called vitamin Bh) in glycerol at ambient temperature $25{ }^{\circ} \mathrm{C}$ could also be achieved. The mechanism could be associated with the friction-induced dissociation of glycerol and interaction of water-like species with the steel surface. This remarkable superlubricity behavior has also recently been seen with other glycerol solution, with acids, or polyhydric alcohol [101, 102].

\section{Physicochemistry process of nanoparticular as lubricant additives}

Nanoparticles have been explored as lubricant additives for about 20 years [103]. With respect to the bulk materials, the nano additives have low melting points (in case of metallic nanoparticles), high boundary adsorption ability, and high reactivity owing to the small size and interfacial effects. Thus, they easily form boundary layers of low shearing strength at the frictional surfaces, to reduce the friction coefficient and to repair the worn surface through deposition at more severe conditions [104]. Xue et al. have proposed the tribochemistry and antiwear mechanism of organic-inorganic nanoparticles, such as the dialkyldithiophosphate coated $\mathrm{Cu}, \mathrm{Ag}, \mathrm{LaF}_{3}$, and the organic acid coated $\mathrm{TiO}_{2}$ nanoparticles as lubricant additives at different lubricating regimes [105]. The schematic lubricating mechanisms are demonstrated 
in Fig. 17 [106]. Under mild sliding conditions, dialkyldithiophosphate and the organic acid can become orientated perpendicular to the specimen surface, and thus, form a film to withstand the local contact pressure. When the lubricating film between the tribo-pairs becomes thinner, and mixed or boundary lubrication occurs, the nanoparticles may carry a proportion of load and separate the two surfaces to prevent adhesion, thus benefiting the antiwear properties. When the shearing is strong, the nanoparticles' core/shell structure may be destroyed and the surface capping layer desorbed and decomposed. The inorganic core may be melted and welded (inert metal nanoparticles such as silver) on the shearing surface, or attached on the surface (adherent, laminar material such as lanthanum trifluoride), or react with the specimen to form a protective layer, thus providing good antiwear and extreme pressure properties. Oil-soluble boron containing compounds are good friction-reducing additives and possess antirust and anticorrosive properties, but they are liable to hydrolyze. Hu et al. have synthesized amorphous lanthanum borate nanoparticles as a wear resistance additive in lubricating oil [107]. The wear resistance and load carrying capacity of base oil was significantly improved. The tribochemical reaction product of FeB plays a key role in antiwear at the boundary lubricating condition.

Layered inorganic nanoparticles such as colloidal molybdenum disulfide, graphite, and boron nitride, have high slip incidence between layers that are
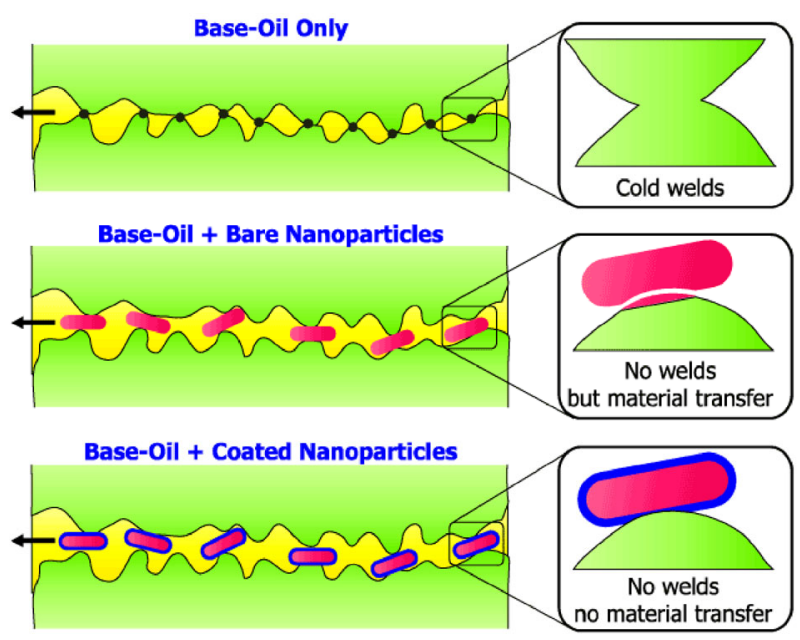

Fig. 17 Action mechanisms of nanoparticle additives [106]. good for lubrication. Tannous et al. investigated the tribological property of the fullerene-(IF)-like $\mathrm{MoS}_{2}$ nanoparticles for different rubbing surfaces (steel, alumina, and diamond-like carbon) [108]. The results indicated that IF-MoS $\mathrm{M}_{2}$ PAO showed good friction reduction and antiwear properties for steel/steel contact. A tribofilm composed of $2 \mathrm{H}-\mathrm{MoS}_{2}$ layers was observed on steel surfaces. It was found that these $\mathrm{MoS}_{2}$ sheets were not only incorporated in the native iron oxide layer present at the outer surface of the steel, but also a reaction occurred between $\mathrm{MoS}_{2}$ and the iron atoms/iron oxide species. $\mathrm{S}-\mathrm{O}, \mathrm{Mo}-\mathrm{O}$, and $\mathrm{Fe}-\mathrm{S}$ bonds were identified by $\mathrm{X}$-ray photoelectron spectroscopy (XPS). $\mathrm{WS}_{2}$ has a similar structure to $\mathrm{MoS}_{2}$ and the $\mathrm{WS}_{2}$ nanoparticles can reduce the friction coefficient by $30 \%-50 \%$ [109]. The addition of $\mathrm{IF}-\mathrm{WS}_{2}$ nanoparticles to the base oils results in up to $50 \%$ reduction in friction coefficient in the mixed lubrication regime.

Graphite has a layered structure and it has been used as lubricant owing to the interlayer slip. The single layer form of graphite, graphene, has received research interest recently, which can elucidate the lubricating mechanisms of graphite. Graphene itself can be used as a green nanoadditive. Eswaraiah et al. studied the tribological properties of graphene as additive in engine oil, and found that at concentration of $0.025 \mathrm{mg} / \mathrm{mL}$, when compared with the base oil, the friction coefficient and wear scar diameter decreased by $80 \%$ and $33 \%$, respectively. This can be attributed to the nanobearing mechanism of graphene in engine oil, and to the ultimate mechanical strength of graphene [110]. The tribological properties of oleic acid-modified graphene as PAO-9 oil additive was investigated by Zhang et al. [111]. The results showed that a lower concentration graphene (0.02-0.06 wt\%) effectively improved the tribological properties of lubricating oils, with friction coefficient and wear scar diameter reduced by $17 \%$ and $14 \%$, respectively. Graphene can form a protective layer on the surface of each steel ball at lower concentrations, which introduces the enhanced antiwear performance. However, as the graphene loading exceeds a critical value, the oil film becomes far more discontinuous, thus degrading the antiwear properties and finally leading to dry friction. 


\section{Conclusions and perspectives}

This critical review deals with physicochemistry at frictional interfaces, and presents the frontier research of friction control and lubrication. It is very clear that interface friction depends on the interplay between lubricating media and substrates. The liquid lubrication section is organized around the traditional Stribeck curve, and points out how surface physicochemistry affects lubrication, and presents the research frontier in each regime. In liquid flow on a solid surface or in hydrodynamic lubrication of liquid film separating sliding contacts, large boundary slips are expected to result in apparent friction reduction by reducing the viscous drag. How the advance of surface science can be utilized to create a stable and large boundary slip is an issue to be further explored. In mixed and boundary lubrication, a condition in which the lubricant film becomes too thin to provide total separation, lubricating oils must completely wet a surface in order to form an effective lubricating film. One way to improve the load-carrying capacity is to strengthen the surface interaction. For rational design, high polarity oils are desirable. ILs represents such an ideal candidate. The application of ILs still faces some issues to be tackled, such as the presence of fluorine content that leads to corrosion, low temperature fluidity, and higher cost. Although there are fluorine-free ILs, it is uncertain whether the substitute ILs have comparable tribological properties, which is of importance because the appropriate triboreaction is a guarantee of good performance.

Surface adsorption is the key for the friction modifiers to assume their function. A pertinent question is whether the research progress of self-assembled monolayers can provide some new insights into designing novel additives. The novel additives must have strong enough adsorption strength (head anchors) and easy-to-shear tails. Another issue, which addresses environmental concerns, is whether we can find equivalent head anchors to replace sulphur/phosphorous containing anchors, such as by creating a multivalent interaction to enhance the interaction strength. The surface adsorption dynamics are another factor to be considered (which might vary for different additives on different substrates), but its correlation with tribological properties is rarely studied.
Biomimicking design of lubricants will be a research focus in the future. It is seemingly more helpful for aqueous lubrication. The question is whether we can find some clues to develop environmentally benign additives for oil lubrication. One apparent clue is that nature's realization of ultra-low lubrication and high load capacity depends not only on interface materials, but also on micro/nano structures. Surface ordered structures will play a key role in drag-reduction of fluid flow and in enhancing the elasto-hydrodynamic effect. Besides, how nature's self-healing capability could be used to design novel lubrication materials and techniques is another area for further research.

Under boundary lubrication condition, the tribology behavior is strongly affected by surface chemisorption and surface tribochemical reactions of additives. The reaction film product can interact with the native oxide layer and sometimes with the crystal structure of the substrate. Understanding the tribochemical reaction mechanism and the structure and composition of reaction products plays a crucial role in understanding the failure mechanism and enabling the industrial development of new lubricant systems. It is still rather difficult to carry out in-situ characterization at single asperity and dynamic surface adsorption at nanometer scale. Very excitingly, we have seen some progress regarding, for instance, the use of TEM and AFM to study the friction process of single asperity in-situ. It is supposed that more advanced surface characterization techniques will be implemented.

One final issue is the question of what to do if the entire liquid lubrication fails and dry friction comes into being. This actually happens very often when a component starts relative motion with very low speed and high load. The most feasible solution is to combine liquid and thin solid film lubrication, so that when liquid lubrication fails, the solid lubricating films will undertake the function of lubrication. It is well known that some soft metallic materials (such as $\mathrm{Au}, \mathrm{Ag}, \mathrm{Cu}$, and their metal sulfides), polymers (polytetrafluoro-ethylene), and carbon materials have low shearing strength. Thus, thin solid films made of these materials can provide low friction coefficient and antiwear properties. By combining liquid and thin solid film lubrication, it is highly possible to realize lubrication at all regimes. The tribophysicochemistry 
between liquid lubricants and thin film materials (rather than conventional steel, alloys, ceramics, etc.) will need more detailed investigation. This research will become increasingly important in the future.

\section{Acknowledgement}

The work is financially supported by the National Natural Science Foundation of China (NSFC) (Nos. 51675512 and 51705504), the National Key Basic Research and Development (973) Program of China (No. 2013CB632301), and Natural Science Foundation of Gansu Province (No. 1606RJZA051).

Open Access: The articles published in this journal are distributed under the terms of the Creative Commons Attribution 4.0 International License (http:// creativecommons.org/licenses/by/4.0/), which permits unrestricted use, distribution, and reproduction in any medium, provided you give appropriate credit to the original author(s) and the source, provide a link to the Creative Commons license, and indicate if changes were made.

\section{References}

[1] Matta C, Joly-Pottuz L, De Barros Bouchet M, Martin J, Kano M, Zhang Q, Goddard W A III. Superlubricity and tribochemistry of polyhydric alcohols. Phys Rev B 78: 085436 (2008)

[2] Cai M R, Guo R S, Zhou F, Liu W M. Lubricating a bright future: Lubrication contribution to energy saving and low carbon emission. Sci China Technol Sci 56: 2888-2913 (2013)

[3] Zhou F, Wu Y. A novel insight into "lubrication". Tribology 36: 132-136 (2016)

[4] Ye C F, Liu W M, Chen Y X, Yu L G. Room-temperature ionic liquids: A novel versatile lubricant. Chem Comm 21: 2244-2245 (2001)

[5] $\mathrm{Mu} \mathrm{Z} \mathrm{G,} \mathrm{Liu} \mathrm{W} \mathrm{M,} \mathrm{Zhang} \mathrm{S.} \mathrm{Functional} \mathrm{room-temperature}$ ionic liquids as lubricants for an aluminum-on-steel system. Chem Lett 33: 524-525 (2004)

[6] Minami I, Inada T, Sasaki R, Nanao H. Tribo-chemistry of phosphonium-derived ionic liquids. Tribol Lett 40: 225-235 (2010)

[7] Liu X Q, Zhou F, Liang Y M, Liu W M. Tribological performance of phosphonium based ionic liquids for an aluminum-on-steel system and opinions on lubrication mechanism. Wear 261: 1174-1179 (2006)
[8] Shah F U, Glavatskih S, MacFarlane D R, Somers A, Forsyth M, Antzutkin O N. Novel halogen-free chelated orthoborate-phosphonium ionic liquids: Synthesis and tribophysical properties. Phys Chem Chem Phys 13: 12865 12873 (2011)

[9] Qu J, Bansal D G, Yu B, Howe J Y, Luo H, Dai S, et al. Antiwear performance and mechanism of an oil-miscible ionic liquid as a lubricant additive. ACS Appl Mater Interfaces 4: 997-1002 (2012)

[10] Forsyth M, Kemp T F, Howlett P C, Sun J Z, Smith M E. A potential novel rapid screening NMR approach to boundary film formation at solid interfaces in contact with ionic liquids. J Phys Chem C 112: 13801-13804 (2008)

[11] Kajdas C. Importance of anionic reactive intermediates for lubricant component reactions with friction surfaces. $\mathrm{Lubr}$ Sci 6: 203-228 (1994)

[12] Mezger M, Schröder H, Reichert H, Schramm S, Okasinski JS, Schöder S, et al. Molecular layering of fluorinated ionic liquids at a charged sapphire (0001) surface. Science 322: 424-428 (2008)

[13] Li H, Wood R J, Rutland M W, Atkin R. An ionic liquid lubricant enables superlubricity to be "switched on" in situ using an electrical potential. Chem Comm 50: 4368-4370 (2014)

[14] Spikes H. Friction modifier additives. Tribol Lett 60: 5 (2015)

[15] Bowden F P, Gregory J N, Tabor D. Lubrication of metal surfaces by fatty acids. Nature 156: 97-101 (1945)

[16] Minami I, Mori S. Concept of molecular design towards additive technology for advanced lubricants. Lubr Sci 19: 127-149 (2007)

[17] Ulman A. Formation and structure of self-assembled monolayers. Chem Rev 96: 1533-1554 (1996)

[18] Ye Q, Zhou F, Liu W M. Bioinspired catecholic chemistry for surface modification. Chem Soc Rev 40: 4244-4258 (2011)

[19] Takahashi K, Shitara Y, Kaimai T, Kanno A, Mori S. Lubricating properties of TR Gel-lube-Influence of chemical structure and content of gel agent. Tribol Int 43: 1577-1583 (2010)

[20] Ohno N, Mia S, Masuhara K, Sonoda K, Yamashita Y, Tamura Y, Morita S, Shitara Y. Tribological properties and film formation behavior of thermoreversible gel lubricants. Tribol Trans 53: 722-730 (2010)

[21] Yu Q L, Fan M J, Li D M, Song Z H, Cai M R, Zhou F, Liu W M. Thermoreversible gel lubricants through universal supramolecular assembly of a nonionic surfactant in a variety of base lubricating liquids. ACS Appl Mater Interfaces 6: 15783-15794 (2014)

[22] Huang G W, Yu Q L, Cai M R, Zhou F, Liu W M. Highlighting the effect of interfacial interaction on tribological 
properties of supramolecular gel lubricants. ACS Appl Mater Interfaces 3 (2016)

[23] Yu Q L, Huang G W, Cai M R, Zhou F, Liu W M. In situ zwitterionic supramolecular gel lubricants for significantly improved tribological properties. Tribol Int 95: 55-65 (2016)

[24] Yu Q L, Li D M, Cai M R, Zhou F, Liu W M. Supramolecular gel lubricants based on amino acid derivative gelators. Tribol Lett 61 (2016)

[25] Cai M R, Liang Y M, Zhou F, Liu W M. Functional ionic gels formed by supramolecular assembly of a novel low molecular weight anticorrosive/antioxidative gelator. J Mater Chem 21: 13399 (2011)

[26] Yu Q L, Wu Y, Li D M, Cai M R, Zhou F, Liu W M. Supramolecular ionogel lubricants with imidazolium-based ionic liquids bearing the urea group as gelator. $J$ Colloid Interf Sci 487: 130-140 (2017)

[27] Wathier M, Lakin B A, Bansal P N, Stoddart S S, Snyder B D, Grinstaff M W. A large-molecular-weight polyanion, synthesized via ring-opening metathesis polymerization, as a lubricant for human articular cartilage. J Am Chem Soc 135: 4930-4933 (2013)

[28] Briscoe W H, Titmuss S, Tiberg F, Thomas R K, McGillivray D J, Klein J. Boundary lubrication under water. Nature 444: 191-194 (2006)

[29] Goldberg R, Schroeder A, Silbert G, Turjeman K, Barenholz $\mathrm{Y}$, Klein J. Boundary lubricants with exceptionally low friction coefficients based on 2D close-packed phosphatidylcholine liposomes. Adv Mater 23: 3517-3521 (2011)

[30] Klein J. Hydration lubrication. Friction 1: 1-23 (2013)

[31] Raviv U, Giasson S, Kampf N, Gohy J F, Jerome R, Klein J. Lubrication by charged polymers. Nature 425: 163-165 (2003)

[32] Lee S, Spencer N D. Materials science. Sweet, hairy, soft, and slippery. Science 319: 575-576 (2008)

[33] Klein J. Repair or peplacement: A joint perspective. Science 323: 47-48 (2009)

[34] Hartung W, Rossi A, Lee S, Spencer N D. Aqueous lubrication of $\mathrm{SiC}$ and $\mathrm{Si}_{3} \mathrm{~N}_{4}$ ceramics aided by a brush-like copolymer additive, poly(l-lysine)-graft-poly(ethylene glycol). Tribol Lett 34: 201-210 (2009)

[35] Perry S S, Yan X, Limpoco F T, Lee S, Muller M, Spencer N D. Tribological properties of poly(L-lysine)graft-poly(ethylene glycol) films: Influence of polymer architecture and adsorbed conformation. ACS Appl Mater Interfaces 1: 1224-1230 (2009)

[36] Heeb R, Bielecki R M, Lee S, Spencer N D. Roomtemperature, aqueous-phase fabrication of poly(methacrylic acid) brushes by UV-LED-induced, controlled radical polymerization with high selectivity for surface-bound species. Macromolecules 42: 9124-9132 (2009)

[37] Chen M, Briscoe W H, Armes S P, Klein J. Lubrication at physiological pressures by polyzwitterionic brushes. Science
323: 1698-1701 (2009)

[38] Wei Q, Cai M, Zhou F, Liu W. Dramatically tuning friction using responsive polyelectrolyte brushes. Macromolecules 46: 9368-9379 (2013)

[39] Nordgren N, Rutland M W. Tunable nanolubrication between dual-responsive polyionic grafts. Nano Lett 9: 2984-2990 (2009)

[40] Zhang R, Ma S H, Wei Q B, Ye Q, Yu B, Gucht J V D, Zhou F. The weak interaction of surfactants with polymer brushes and its impact on lubricating behavior. Macromolecules 48: 6186-6196 (2015)

[41] Kyomoto M, Moro T, Saiga K, Miyaji F, Kawaguchi H, Takatori Y, Nakamura K, Ishihara K. Lubricity and stability of poly(2-methacryloyloxyethylphosphorylcholine) polymer layer on Co-Cr-Mo surface for hemi-arthroplasty to prevent degeneration of articular cartilage. Biomaterials 31: 658-668 (2010)

[42] Kyomoto M, Moro T, Saiga K, Hashimoto M, Ito H, Kawaguchi H, Takatori Y, Ishihara K. Biomimetic hydration lubrication with various polyelectrolyte layers on cross-linked polyethylene orthopedic bearing materials. Biomaterials 33: 4451-4459 (2012)

[43] Kobayashi M, Terada M, Takahara A. Polyelectrolyte brushes: A novel stable lubrication system in aqueous conditions. Faraday Discussions 156: 403-412 (2012)

[44] Philippon D, De Barros-Bouchet M I, Le Mogne T, Lerasle O, Bouffet A, Martin J M. Role of nascent metallic surfaces on the tribochemistry of phosphite lubricant additives. Tribol Int 44: 684-691 (2011)

[45] Hsu S M, Zhang J, Yin Z F. The nature and origin of tribochemistry. Tribol Lett 13: 131-139 (2002)

[46] Nakayama K. Triboemission of electrons, ions, and photons from diamondlike carbon films and generation of tribomicroplasma. Surface \& Coatings Technology 188: 599-604 (2004)

[47] Nakayama K. Effect of magnetic field on the plasma generated during a sliding contact. J Phys: Conf Ser 2011: 012069 (2011)

[48] Nakayama K. Mechanism of triboplasma generation in oil. Tribol Lett 41: 345-351 (2011)

[49] Nakayama K, Hashimoto H. Triboemission, tribochemical reaction, and friction and wear in ceramics under various n-butane gas pressures. Tribol Int 29: 385-393 (1996)

[50] Nakayama K, Martin J M. Tribochemical reactions at and in the vicinity of a sliding contact. Wear 261: 235-240 (2006)

[51] Spikes H. The history and mechanisms of ZDDP. Tribol Lett 17: 469-489 (2004)

[52] Topolovec-Miklozic K, Forbus T R, Spikes H A. Film thickness and roughness of ZDDP antiwear films. Tribol Lett 26: 161-171 (2007)

[53] Mosey N J. Molecular mechanisms for the functionality of lubricant additives. Science 307: 1612-1615 (2005) 
[54] Gosvami N N, Bares J A, Mangolini F, Konicek A R, Yablon D G, Carpick R W. Mechanisms of antiwear tribofilm growth revealed in situ by single-asperity sliding contacts. Science 348: 102-106 (2015)

[55] Morina A, Neville A, Priest M, Green J H. ZDDP and MoDTC interactions and their effect on tribological performance - tribofilm characteristics and its evolution. Tribol Lett 24: 243-256 (2006)

[56] Grossiord C, Varlot K, Martin J-M, Th. Le Mogne C E, Inoue $\mathrm{K} . \mathrm{MoS}_{2}$ single sheet lubrication by molybdenum dithiocarbamate. Tribol Int 31: 737-747 (1998)

[57] Shah F U, Glavatskih S, Höglund E, Lindberg M, Antzutkin $\mathrm{O}$ N. Interfacial antiwear and physicochemical properties of alkylborate-dithiophosphates. ACS Appl Mater Interfaces 3: 956-968 (2011)

[58] Zhou F, Liang Y M, Liu W M. Ionic liquid lubricants: Designed chemistry for engineering applications. Chem Soc Rev 38: 2590-2599 (2009)

[59] Huang G W, Yu Q L, Cai M R, Zhou F, Liu W M. Investigation of the lubricity and antiwear behavior of guanidinium ionic liquids at high temperature. Tribol Int 114: $65-76$ (2017)

[60] Zhang S W, Hu L T, Qiao D, Feng D P, Wang H Z. Vacuum tribological performance of phosphonium-based ionic liquids as lubricants and lubricant additives of multialkylated cyclopentanes. Tribol Int 66: 289-295 (2013)

[61] Fan M J, Yang D S, Wang X L, Liu W M, Fu H Z. DOSSbased QAILs: As both neat lubricants and lubricant additives with excellent tribological properties and good detergency. Ind Eng Chem Res 53: 17952-17960 (2014)

[62] Otero I, Lopez E R, Reichelt M, Villanueva M, Salgado J, Fernandez J. Ionic liquids based on phosphonium cations as neat lubricants or lubricant additives for a steel/steel contact. ACS Appl Mater Interfaces 6: 13115-13128 (2014)

[63] Qiao D, Wang H Z, Feng D P. Tribological performance and mechanism of phosphate ionic liquids as additives in three base oils for steel-on-aluminum contact. Tribol Lett 55: 517-531 (2014)

[64] Anand M, Hadfield M, Viesca J L, Thomas B, Battez A H, Austen S. Ionic liquids as tribological performance improving additive for in-service and used fully-formulated diesel engine lubricants. Wear 334: 67-74 (2015)

[65] Fu X S, Sun L G, Zhou X G, Li Z P, Ren T H. Tribological study of oil-miscible quaternary ammonium phosphites ionic liquids as lubricant additives in PAO. Tribol Lett 60: 23 (2015)

[66] Qu J, Barnhill W C, Luo H M, Meyer H M, 3rd, Leonard D N, Landauer A K, Kheireddin B, Gao H, Papke B L, Dai S. Synergistic effects between phosphonium-alkylphosphate ionic liquids and zinc dialkyldithiophosphate (ZDDP) as lubricant additives. Adv Mater 27: 4767-4774 (2015)
[67] Qu J, Meyer H M, III, Cai Z B, Ma C, Luo H M. Characterization of ZDDP and ionic liquid tribofilms on non-metallic coatings providing insights of tribofilm formation mechanisms. Wear 332: 1273-1285 (2015)

[68] Li H, Somers A E, Howlett P C, Rutland M W, Forsyth M, Atkin R. Addition of low concentrations of an ionic liquid to a base oil reduces friction over multiple length scales: A combined nano- and macrotribology investigation. Phys Chem Chem Phys 18: 6541-6547 (2016)

[69] Huang G W, Yu Q L, Ma Z F, Cai M R, Liu W M. Probing the lubricating mechanism of oil-soluble ionic liquids additives. Tribol Int 107: 152-162 (2017)

[70] Zhou Y, Qu J. Ionic liquids as lubricant additives: a review. ACS Appl Mater Interfaces 9: 3209-3222 (2017)

[71] Gusain R, Gupta P, Saran S, Khatri O P. Halogen-free bis(imidazolium)/bis(ammonium)di bis(salicylato)borate ionic liquids as energy-efficient and environmentally friendly lubricant additives. ACS Appl Mater Inter 6: 15318-15328 (2014)

[72] Gusain R, Singh R, Sivakumar K L N, Khatri O P. Halogen-free imidazolium/ammonium-bis(salicylato)borate ionic liquids as high performance lubricant additives. RSC Adv 4: 1293-1301 (2014)

[73] Taher M, Shah F U, Filippov A, de Baets P, Glavatskih S, Antzutkin $\mathrm{O}$ N. Halogen-free pyrrolidinium bis(mandelato)borate ionic liquids: Some physicochemical properties and lubrication performance as additives to polyethylene glycol. RSC Adv 4: 30617-30623 (2014)

[74] Khatri P K, Joshi C, Thakre G D, Jain S L. Halogen-free ammonium-organoborate ionic liquids as lubricating additives: The effect of alkyl chain lengths on the tribological performance. New J Chem 40: 5294-5299 (2016)

[75] Gusain R, Bakshi P S, Panda S, Sharma O P, Gardas R, Khatri O P. Physicochemical and tribophysical properties of trioctylalkylammonium bis(salicylato)borate (N888n-BScB) ionic liquids: Effect of alkyl chain length. Phys Chem Chem Phys 19: 6433-6442 (2017)

[76] Minami I, Kamimura H, Mori S. Thermo-oxidative stability of ionic liquids as lubricating fluids. J Synth Lubr 24: 135-147 (2007)

[77] Minami I. Ionic liquids in tribology. Molecules 14: 2286-2305 (2009)

[78] Cai M R, Liang Y M, Zhou F, Liu W M. Anticorrosion imidazolium ionic liquids as the additive in poly(ethylene glycol) for steel/Cu-Sn alloy contacts. Faraday Discuss 156: 147-157 (2012)

[79] Cai M R, Liang Y M, Zhou F, Liu W M. Tribological properties of novel imidazolium ionic liquids bearing benzotriazole group as the antiwear/anticorrosion additive in poly(ethylene glycol) and polyurea grease for steel/steel Contacts. ACS Appl Mater Interfaces 3: 4580-4592 (2011) 
[80] Cai M R, Liang Y M, Yao M H, Xia Y Q, Zhou F, Liu W M. Imidazolium ionic liquids as antiwear and antioxidant additive in poly(ethylene glycol) for steel/steel contacts. Acs Appl Mater Interfaces 2: 870-876 (2010)

[81] Cai M R, Liang Y M, Zhou F, Liu W M. A novel imidazolium salt with antioxidation and anticorrosion dual functionalities as the additive in poly(ethylene glycol) for steel/steel contacts. Wear 306: 197-208 (2013)

[82] Song Z H, Cai M R, Liang Y M, Fan M J, Zhou F, Liu W M. In situ preparation of anti-corrosion ionic liquids as the lubricant additives in multiply-alkylated cyclopentanes. Rsc $A d v$ 3: 21715-21721 (2013)

[83] Song Z H, Liang Y M, Fan M J, Zhou F, Liu W M. Lithiumbased ionic liquids as novel lubricant additives for multiply alkylated cyclopentanes (MACs). Friction 2: 392 (2014)

[84] Fan M, Liang Y, Zhou F, Liu W. Dramatically improved friction reduction and wear resistance by in situ formed ionic liquids. Rsc Adv 2: 6824-6830 (2012)

[85] Wang Y R, Yu Q L, Cai M R, Shi L, Zhou F, Liu W M. Synergy of lithium salt and non-ionic surfactant for significantly improved tribological properties of water-based fluids. Tribol Int 113: 58-64 (2016)

[86] Fan M J, Song Z H, Liang Y M, Zhou F, Liu W M. In situ formed ionic liquids in synthetic esters for significantly improved lubrication. ACS Appl Mater Interfaces 4: 66836689 (2012)

[87] Hirano M. Superlubricity: A state of vanishing friction. Wear 254: 932-940 (2003)

[88] Chhowalla M, Amaratunga G A J. Thin films of fullerenelike $\mathrm{MoS}_{2}$ nanoparticles with ultra-low friction and wear. Nature 407: 164-167 (2000)

[89] Heimberg J A, Wahl K J, Singer I L, Erdemir A. Superlow friction behavior of diamond-like carbon coatings: time and speed effects. Appl Phys Lett 78: 2449 (2001)

[90] Li H, Wang J, Gao S, Chen Q, Peng L, Liu K, Wei X. Superlubricity between $\mathrm{MoS}_{2}$ monolayers. Adv Mater 29: 1701474 (2017)

[91] Li J, Zhang C, Luo J. Superlubricity behavior with phosphoric acid-water network induced by rubbing. Langmuir $\mathbf{2 7}$ 9413-9417 (2011)

[92] Li J, Luo J. Advancements in superlubricity. Sci China Technol Sci 56: 2877-2887 (2013)

[93] Li J J, Zhang C H, Deng M M, Luo J B. Investigations of the superlubricity of sapphire against ruby under phosphoric acid lubrication, Friction 2: 164-172 (2014)

[94] Zeng Q F, Dong G N, Martin J M. Green superlubricity of nitinol 60 alloy against steel in presence of castor oil. $A d v$ Mater Interfaces 6: 29992 (2016)

[95] Zhang C X, Liu Z F, Liu Y H, Cheng Q, Yang C B, Cai L G. Investigation of the mechanisms for stable superlubricity of poly(vinylphosphonic acid) (PVPA) coatings affected by lubricant. Friction 4: 303-312 (2016)
[96] Zhang C-H, Ma Z-Z, Luo J-B, Lu X-C, Wen S-Z. Superlubricity of a mixed aqueous solution. Chinese Phys Lett 28: 056201 (2011)

[97] Li J J, Zhang C H, Cheng P, Chen X C, Wang W Q, Luo J B. AFM Studies on liquid superlubricity between silica surfaces achieved with surfactant micelles. Langmuir 32: 5593-5599 (2016)

[98] Li J, Ma L, Zhang S, Zhang C, Liu Y, Luo J. Investigations on the mechanism of superlubricity achieved with phosphoric acid solution by direct observation. J Appl Phys 114: 114901 (2013)

[99] Li J, Zhang C, Sun L, Lu X, Luo J. Tribochemistry and superlubricity induced by hydrogen ions. Langmuir 28: 15816-15823 (2012)

[100] Yue D-C, Ma T-B, Hu Y-Z, Yeon J, van Duin A C T, Wang H, Luo J B. Tribochemistry of phosphoric acid sheared between quartz surfaces: A reactive molecular dynamics study. J Phys Chem C 117: 25604-25614 (2013)

[101] Li J, Zhang C, Luo J. Superlubricity achieved with mixtures of polyhydroxy alcohols and acids. Langmuir 29: 5239 5245 (2013)

[102] Li J, Zhang C, Ma L, Liu Y, Luo J. Superlubricity achieved with mixtures of acids and glycerol. Langmuir 29: 271275 (2013)

[103] Zhang Z J, Zhang J, Xue Q J. Synthesis and characterization of a molybdenum disulfide nanoclustert. J Phys Chem 98: 12973-12977 (1994)

[104] Liu W M, Wang X B. Nanolubricants made of metals. In Nanolubricants. Martinc J M, Ed. England: John Wiley \& Sons, 2008: 175-201 (2008)

[105] Li B, Wang X, Liu W, Xue Q. Tribochemistry and antiwear mechanism of organic-inorganic nanoparticles as lubricant additives. Tribol Lett 22: 79-84 (2006)

[106] Akbulut M. Nanoparticle-based lubrication systems. J Powder Metall Min 1: 1000e1101 (2012)

[107] Hu Z S, Dong J X, Chen G X, He J Z. Preparation and tribological properties of nanoparticle lanthanum borate. Wear 243: 43-47 (2000)

[108] Tannous J, Dassenoy F, Lahouij I, Mogne T, Vacher B, Bruhács A, Tremel W. Understanding the tribochemical mechanisms of IF- $\mathrm{MoS}_{2}$ nanoparticles under boundary lubrication. Tribol Lett 41: 55-64 (2010)

[109] Greenberg R, Halperin G, Etsion I, Tenne R. The effect of $\mathrm{WS}_{2}$ nanoparticles on friction reduction in various lubrication regimes. Tribol Lett 17: 179-186 (2004)

[110] Eswaraiah V, Sankaranarayanan V, Ramaprabhu S. Graphenebased engine oil nanofluids for tribological applications. ACS Appl Mater Interfaces 3: $4221-4227$ (2011)

[111] Zhang W, Zhou M, Zhu H, Tian Y, Wang K, Wei J, Ji F, Li X, Li Z, Zhang P, Wu D. Tribological properties of oleic acid-modified graphene as lubricant oil additives. J Phys D: Appl Phys 44: 205303 (2011) 


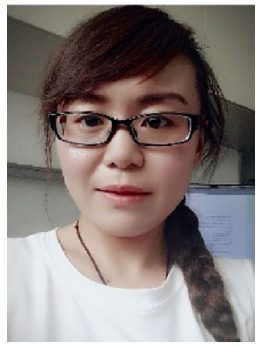

Meirong CAI. She got her $\mathrm{PhD}$ degree in 2012 at the Lanzhou Institute of Chemical Physics. She is an associate professor at the State Key Lab of Solid Lubrication in

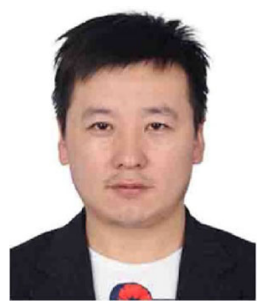

Qiangliang YU. He got his $\mathrm{PhD}$ degree in 2016 at the Lanzhou Institute of Chemical Physics. He is an assistant at the State Key Lab of

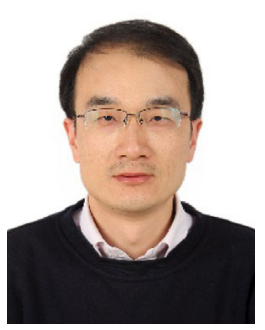

Feng ZHOU. He is a full professor in Lanzhou Institute of Chemical Physics, CAS and deputy director of State Key Laboratory of Solid Lubrication. He gained PhD degree in 2004 and spent three years (2005-2008) in the Department of Chemistry, University of Cambridge as a research associate. He has published more than 300 journal papers that received more than 10,000 citations and

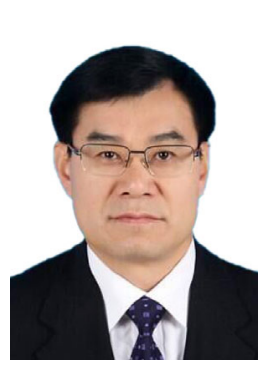

Weimin LIU. He received his Ph.D. degree in lubricating materials and tribology from Lanzhou Institute of Chemical Physics (LICP) of the Chinese Academy of Sciences in 1990. After that, he joined the State Key Laboratory of Solid Lubrication (LSL) of the LICP. From June 1993 to June 1994, he worked as a visiting scholar at Pennsylvania State University, USA. In 2013, he was elected the Member of the Chinese Academy of
Lanzhou Institute of Chemical Physics, CAS. She has authored or co-authored more than 40 journal papers. Her research interests are ionic liquids lubricants and supramolecular gel lubricants.

Solid Lubrication in Lanzhou Institute of Chemical Physics, CAS. His research interests are ionic liquids lubricants and anticorrosive additives.

has the $\mathrm{H}$-index 60 . His research interests include the bioninspired tribology, biomimic surfaces/interfaces of soft matters, drag-reduction and antibiofouling, and functional coatings. He has gained a number of awards including "Outstanding Youth Award" of International Society of Bionic Engineering, 2013. He serves as editorial board member of Tribology International, Journal Fiber Bioengineering and Informatics, etc.

Sciences. Currently, he is a professor of LICP and head of the State Key Laboratory of Solid Lubrication. Up to now, he has published more than 500 papers with citation over 20,000. He holds 80 Chinese patents and 1 US patent; won 2 National Awards for Technological Invention (second class) and 1 National Award for Natural Sciences (second class). Currently, his research interests mainly focus on space and aviation lubrication, high performance lubricating materials, and tribochemistry. 